\title{
COHOMOLOGY OF TILTING MODULES OVER QUANTUM GROUPS AND $t$-STRUCTURES ON DERIVED CATEGORIES OF COHERENT SHEAVES
}

\author{
ROMAN BEZRUKAVNIKOV
}

To the memory of my father.

\begin{abstract}
The paper is concerned with cohomology of the small quantum group at a root of unity, and of its upper triangular subalgebra, with coefficients in a tilting module. It turns out to be related to irreducible objects in the heart of a certain $t$-structure on the derived category of equivariant coherent sheaves on the Springer resolution, and to equivariant coherent IC sheaves on the nil-cone. The support of the cohomology is described in terms of cells in affine Weyl groups. The basis in the Grothendieck group provided by the cohomology modules is shown to coincide with the Kazhdan-Lusztig basis, as predicted by J. Humphreys and V. Ostrik.

The proof is based on the results of $[\mathrm{ABG}],[\mathrm{AB}]$ and $[\mathrm{B}]$, which allow us to reduce the question to purity of IC sheaves on affine flag varieties.
\end{abstract}

\section{Contents}

1. Introduction.

1.1. Quantum groups, tilting modules and cohomology.

1.2. Koszul duality conjecture.

1.3. Plan of the paper.

1.4. Notations.

1.5. Acknowledgements.

2. Coherent sheaves: exotic $t$-structure, and Positivity Lemma.

2.1. Exceptional sets and quasi-hereditary hearts. $\quad 6$

2.2. Sheaves on the nilpotent cone. 11

2.3. Exotic $t$-structure. $\quad 12$

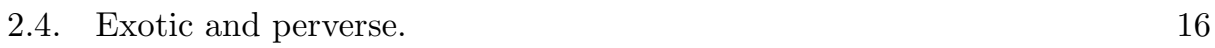

2.5. Positivity Lemma. 17

3. Quantum group modules: proof modulo the Positivity Lemma. 18

$\begin{array}{lll}3.1 . & \text { Some results of }[\mathrm{ABG}] . & 18\end{array}$

3.2. Consequences of Theorem 3. 19

3.3. Proof of Theorem 3 modulo the Positivity Lemma. 19

4. Constructible sheaves on affine flags: proof of Positivity Lemma. 20

4.1. Perverse sheaves on affine flags for $G^{`}$. 20

4.2. Frobenius weights. 23

4.3. Proof of the Positivity Lemma. 25

References 26 


\section{InTRODUCTION.}

1.1. Quantum groups, tilting modules and cohomology. Let $G$ be a semisimple complex algebraic group of adjoint type, $N \subset B \subset G$ be a maximal unipotent and a Borel subgroups, and $\mathfrak{n} \subset \mathfrak{b} \subset \mathfrak{g}$ be the corresponding Lie algebras. Let $\mathcal{N} \subset \mathfrak{g}$ be the nilpotent cone, let $\tilde{\mathcal{N}}=T^{*}(G / B)=G \times_{B} \mathfrak{n}=\{(g B, x) \mid x \in \operatorname{Ad}(g)(\mathfrak{n})\}$ and let $\pi: \tilde{\mathcal{N}} \rightarrow \mathcal{N}$ be the Springer-Grothendieck map. For an algebraic group $H$ acting on an algebraic variety $X$ we let $\operatorname{Coh}^{H}(X)$ denote the category of $H$ equivariant coherent sheaves on $X$, and $D^{H}(X)=D^{b}\left(\operatorname{Coh}^{H}(X)\right)$ be the bounded derived category.

Let $q \in \mathbb{C}$ be a primitive root of unity of order $l$. We assume that $l$ is odd, and prime to 3 if $\mathfrak{g}$ has a factor of type $G_{2}$; and also that $l$ is greater than the Coxeter number. Let $\mathrm{U}_{q}$ be the Lusztig quantum enveloping algebra [Lq]. We have finite dimensional subalgebras $b_{q} \subset u_{q} \subset \mathrm{U}_{q}$, where $u_{q}$ is the so-called "small quantum group", and $b_{q} \subset u_{q}$ is the upper triangular subalgebra.

For an augmented $\mathbb{C}$-algebra $A$ we write $H^{\bullet}(A)=\operatorname{Ext}_{A}(\mathbb{C}, \mathbb{C})$, and $H^{\bullet}(A, M)=$ $\operatorname{Ext}_{A}(\mathbb{C}, M)$ for an $A$ module $M$; thus $H^{\bullet}(A)$ is a graded associative algebra, and $H^{\bullet}(A, M)$ is a graded module over this algebra.

We consider the category $\mathrm{U}_{q}$-mod of finite dimensional graded $\mathrm{U}_{q}$ modules; we let $\mathrm{U}_{q}$ - $\bmod ^{0} \subset \mathrm{U}_{q}$-mod be the block of the trivial module, see, e.g., [ABG], $\S 3.4$ (cf. also $[\mathrm{AG}], \S 1.2)$.

According to [GK] we have $H^{\bullet}\left(u_{q}\right) \cong \mathcal{O}(\mathcal{N})$. Thus for a $u_{q}$ module $M$ we get an $H^{\bullet}\left(u_{q}\right)=\mathcal{O}(\mathcal{N})$ module $H(M)=H^{\bullet}\left(u_{q}, M\right)$. Moreover, it is explained in [GK] that if $M$ is an $\mathrm{U}_{q}$ module then the graded $\mathcal{O}(\mathcal{N})$ module $H(M)$ is $G$-equivariant, thus we get a functor $H: \mathrm{U}_{q}-\bmod \rightarrow \operatorname{Coh}^{G \times \mathbb{G}_{m}}(\mathcal{N}), H(M)=H^{\bullet}(M)$, where the multiplicative group $\mathbb{G}_{m}$ acts on $\mathcal{N}$ by $t: x \mapsto t^{2} x$.

In this paper we provide a reasonably explicit description of $H(M)$ when $M$ is a tilting object of $\mathrm{U}_{q}-\bmod ^{0}$. To state this description we have to recall the category $\mathcal{P} C$ oh of $G$-equivariant coherent perverse sheaves on $\mathcal{N}$ with respect to the middle perversity, see [B1]. It is the heart of a certain $t$-structure on $D^{G}(\mathcal{N})$. For each pair $(O, \mathcal{L})$ where $O \subset \mathcal{N}$ is a $G$ orbit, and $\mathcal{L}$ is an irreducible $G$-equivariant vector bundle on $O$ there is a unique irreducible object $I C_{O, \mathcal{L}}$ supported on the closure of $O$, and satisfying $\left.I C_{O, \mathcal{L}}\right|_{O}=\mathcal{L}\left[-\frac{\operatorname{codim} O}{2}\right]$; these are all of the irreducible objects of $\mathcal{P C o h}$. We will prove the following

Theorem 1. Let $T$ be an indecomposable tilting object of $\mathrm{U}_{q}$-mod ${ }^{0}$. Then either $H(T)=0$, or there exists a (n obviously unique) pair $\left(O_{T}, \mathcal{L}_{T}\right)$ as above, such that we have an isomorphism of $G$-equivariant $\mathcal{O}(\mathcal{N})$ modules $H(T) \cong \Gamma\left(I C_{O_{T}, \mathcal{L}_{T}}\right)$; here $\Gamma=\bigoplus_{i} R^{i} \Gamma$ denotes the total cohomology (derived global sections) functor.

See Corollary 2 in section 3.2 for a more precise statement. The latter implies, via a result of $[\mathrm{B}]$, a conjecture of J. Humphreys (proved for type $A$ in Ostrik's thesis, see [O1]), which describes the support of $H(T)$ in terms of 2-sided cells in the affine Weyl group (Corollary 3 in section 3.2). Our results also yield some conjectures by V. Ostrik, see Remark 8 . These applications have partly motivated the present work.

In fact, the above statements will be derived from the following stronger results.

First of all, besides of the objects $H(T) \in \operatorname{Coh}^{G}(\mathcal{N})$ we will also describe (though somewhat less explicitly) the objects $\widetilde{H}(T) \in \operatorname{Coh}^{G}(\tilde{\mathcal{N}})$ carrying more information. 
These are defined as follows. By $[\mathrm{GK}]$ we have $H^{\bullet}\left(b_{q}\right)=\mathcal{O}(\mathfrak{n})=\operatorname{Sym}\left(\mathfrak{n}^{*}\right)$; thus for a $b_{q}$ module $M$ we get an $\mathcal{O}(\mathfrak{n})$ module $H^{\bullet}\left(b_{q}, M\right)$. If $M$ is equipped with an action of the Borel subalgebra $\mathrm{B}_{q} \subset \mathrm{U}_{q}$ then the $\mathcal{O}(\mathfrak{n})$ module $H^{\bullet}\left(b_{q}, M\right)$ is equivariant with respect to the adjoint action of the Lie algebra $\mathfrak{b}$. If $M$ is one dimensional, an explicit calculation (see [GK]) shows that this module is locally finite; it follows that the same is true for any complex $M$ of $\mathrm{B}_{q}$ modules whose cohomology is finite dimensional. Thus for such $M$ the $\mathfrak{n}$ action on $H^{\bullet}\left(b_{q}, M\right)$ integrates to an action of the algebraic group $N$; furthermore, if $M$ admits a grading by weights compatible with the $\mathrm{B}_{q}$ action, then the action of $\mathfrak{b}$ integrates to an action of $B$, so we get a functor from the bounded derived category of finite dimensional graded $\mathrm{B}_{q}$ modules to $\operatorname{Coh}^{B}(\mathfrak{n})$. Composing it with the restriction functor from $\mathrm{U}_{q}$ to $\mathrm{B}_{q}$ we get a functor $\widetilde{H}: \mathrm{U}_{q}$-mod $\rightarrow \operatorname{Coh}^{B}(\mathfrak{n}) \cong \operatorname{Coh}^{G}(\tilde{\mathcal{N}})$, where the last equivalence is the induction functor, inverse to $\left.\mathcal{F} \mapsto \mathcal{F}\right|_{\mathfrak{n}}$ (recall that $\tilde{\mathcal{N}}=G \times_{B} \mathfrak{n}$ ).

The description of $\widetilde{H}(T)$ for a tilting object $T \in \mathrm{U}_{q}-\bmod ^{0}$ is as follows. We define a $t$-structure on the bounded derived categories $D^{G \times \mathbb{G}_{m}}(\tilde{\mathcal{N}}), D^{G}(\tilde{\mathcal{N}})$, which we call the exotic t-structure; we call objects of its heart exotic sheaves. We prove that the sheaves $\tilde{H}(T)$, where $T$ is an indecomposable tilting object of $\mathrm{U}_{q}$-mod ${ }^{0}$, are precisely the cohomology sheaves of irreducible exotic sheaves. It turns out that for an irreducible exotic sheaf $E$ the object $R \pi_{*}(E)$ is either zero, or isomorphic to $I C_{O, \mathcal{L}}$ for some $(O, \mathcal{L})$. This allows one to deduce Theorem 1.

Actually, all the above results about cohomology of $\mathrm{U}_{q}$ modules will be deduced from stronger statements involving isomorphisms of objects in the derived category of coherent sheaves. To state the latter we recall that (the first part of) [ABG] provides a triangulated functor $\Psi: D^{G \times \mathbb{G}_{m}}(\tilde{\mathcal{N}}) \rightarrow D^{b}\left(\mathrm{U}_{q}-\bmod ^{0}\right)$ which is "almost an equivalence" (see (18) below for a precise statement), here $\mathbb{G}_{m}$ acts on $\tilde{\mathcal{N}}$ by $t:(g B, x) \mapsto\left(g B, t^{2} x\right)$.

The following statement is the central point of the present paper, see Theorem 3 below for a more precise statement.

Theorem 2. For any irreducible exotic sheaf $E \in D^{G \times \mathbb{G}_{m}}(\tilde{\mathcal{N}})$ we have $\Psi(E) \cong$ $T[n]$ for some indecomposable tilting object $T \in \mathrm{U}_{q}-\bmod ^{0}$ and $n \in \mathbb{Z}$. Every indecomposable tilting $T \in \mathrm{U}_{q}$-mod ${ }^{0}$ has the form $\Psi(E)$ for a unique irreducible exotic sheaf $E \in D^{G \times \mathbb{G}_{m}}(\tilde{\mathcal{N}})$.

This will be deduced from purity of irreducible perverse sheaves on the affine flag variety for the Langlands dual group via results of $[\mathrm{AB}]$.

1.2. Koszul duality conjecture. In this section we attempt to explain the origin of our method.

To clarify the idea behind the argument we gather together various functors, some of which are used in this paper:

$$
D^{b}\left(\mathcal{P}_{s p h}\right) \underset{(I)}{\cong} D^{b}\left(\mathrm{U}_{q}-m o d^{0}\right) \underset{(\bar{I} I)}{\cong} D G C o h^{G}(\tilde{\mathcal{N}}) \stackrel{(I I I)}{\longleftarrow} D^{G \times \mathbb{G}_{m}}(\tilde{\mathcal{N}}) \stackrel{(I V)}{\longrightarrow} D^{G}(\tilde{\mathcal{N}}) \underset{(V)}{\cong} D^{b}\left(\mathcal{P}_{a s p h}\right)
$$

Here $D^{b}\left(\mathcal{P}_{s p h}\right)$ (where "sph" stands for "spherical") is the category of perverse sheaves on the affine Grassmannian for the Langlands dual group $G^{\sim}$ smooth along the Schubert stratification; the equivalence (I) is defined in [ABG]. Unlike the other functors appearing above, this equivalence comes from an equivalence of abelian categories. Neither (I), nor the category $\mathcal{P}_{\text {sph }}$ are used in the present paper, they 
are mentioned here to clarify the picture; thus we will use only the first, "algebraic" part of $[\mathrm{ABG}]$.

The equivalence (II) is also introduced in $[\mathrm{ABG}]$. The category $D G \operatorname{Coh}^{G}(\tilde{\mathcal{N}})$ here is the (derived) category of DG modules over a certain DG algebra with zero differential, such that the category of modules over its cohomology algebra is naturally identified with $\operatorname{Coh}^{G}(\tilde{\mathcal{N}})$. We do not go into details here, referring the interested reader to $[\mathrm{ABG}]$. Instead we use the composition of functors (III) and (II). We remark that providing the composite functor $D^{G \times \mathbb{G}_{m}}(\tilde{\mathcal{N}}) \rightarrow D^{b}\left(\mathrm{U}_{q}-\bmod ^{0}\right)$ satisfying properties (17), (18) below is essentially equivalent to providing the equivalence (II).

(IV) is the forgetful functor, restricting the equivariance from $G \times \mathbb{G}_{m}$ to $G$.

Finally, $D^{b}\left(\mathcal{P}_{a s p h}\right)$ (where "asph" stands for "anti-spherical") is a certain category of perverse sheaves on the affine flag variety of the Langlands dual group $G^{\sim}$, and $(\mathrm{V})$ is proved in $[\mathrm{AB}]$ (see section 4 below for more details). This equivalence will be used in order to deduce the key Positivity Lemma (Lemma 9 in section 2.5) below from purity for perverse sheaves on the affine flag variety.

The shortest (though, perhaps, the least elementary) description of the exotic $t$-structure on $D^{G}(\tilde{\mathcal{N}})$ is as follows: it is the transport of the tautological $t$-structure on $D^{b}\left(\mathcal{P}_{\text {asph }}\right)$ by means of the equivalence $(\mathrm{V})$; the exotic $t$-structure on $D^{G \times \mathbb{G}_{m}}(\tilde{\mathcal{N}})$ is then the unique $t$-structure compatible with the exotic $t$-structure on $D^{G}(\tilde{\mathcal{N}})$. Thus an irreducible exotic sheaf goes to an irreducible object of $D^{b}\left(\mathcal{P}_{a s p h}\right)$ under the composition $(\mathrm{V}) \circ(\mathrm{IV})$. Comparing this with Theorem 2 we discover a relation between tilting objects in $\mathcal{P}_{\text {asph }}$ and irreducible objects in $\mathcal{P}_{\text {sph }}$. This motivates the following conjecture, which inspired the methods of the present work.

To state the conjecture we need the following concept. We will say that two abelian categories $\mathcal{A}$ and $\mathcal{B}$ are derived Koszul equivalent if there exist graded versions ${ }^{1} \mathcal{A}^{g r}, \mathcal{B}^{g r}$ and an equivalence of derived categories $\kappa: D^{b}\left(\mathcal{A}^{g r}\right) \cong D^{b}\left(\mathcal{B}^{g r}\right)$, which satisfies $\kappa(M(1)) \cong \kappa(M)(1)[1]$, where $M \mapsto M(1)$ is the shift of grading functor. The interested reader is referred to [BGS] for examples and comments.

Conjecture. The category of perverse sheaves on the affine flag variety of a semi-simple group, which are smooth along the Schubert stratification is derived Koszul equivalent to itself; the corresponding equivalence interchanges irreducible and tilting objects. There exists also a parabolic - singular version (in the sense of [BGS]) of this duality, whose particular case is a derived Koszul equivalence between $\mathcal{P}_{\text {sph }}$ and $\mathcal{P}_{\text {asph }}$; the corresponding equivalence interchanges tilting and irreducible objects.

This Conjecture is an affine analogue of the main results of [S], [BGS], or rather of a variant of the latter provided by [BG].

In fact, existence of the derived Koszul equivalence between $\mathcal{P}_{\text {sph }}$ and $\mathcal{P}_{\text {asph }}$ sending irreducibles to tiltings follows from the above mentioned results of $[\mathrm{AB}]$, $[\mathrm{ABG}]$, and the present paper. It is also plausible that the first part of the conjecture can be proven by the methods of [S] complemented by the geometric counterpart of translation functors. The latter is a certain collection of exact endofunctors of the category of unipotently monodromic perverse sheaves on the basic affine space of a Kac-Moody group, which in the case of a finite dimensional group reduces to the

\footnotetext{
${ }^{1}$ See, e.g., [BGS], $\S 4.3$ for the definition.
} 
usual reflection functors. They can be defined as convolution with tilting sheaves (cf. [BG], Theorem 6.10); some other constructions will appear in a forthcoming paper by I. Mirković.

Finally, let us mention that the exotic $t$-structure on the derived category of coherent sheaves on $\tilde{\mathcal{N}}$ appears in some other contexts, e.g., it is related to the $t$-structures appearing in [BMR], and, conjecturally, ${ }^{2}$ to modules over the affine Lie algebra on the critical level studied by Frenkel and Gaitsgory, cf. [FG].

1.3. Plan of the paper. In section 2, after some preliminaries about mutations of exceptional sets and $t$-structures defined by generating exceptional sets, we define the exotic $t$-structure on $D^{G}(\tilde{\mathcal{N}}), D^{G \times \mathbb{G}_{m}}(\tilde{\mathcal{N}})$; we also relate it to the middle perversity $t$-structure on $D^{G}(\mathcal{N})$. We finish the section by stating the Positivity Lemma. In section 3 , modules over the quantum group at a root of unity appear: we recall the results of $[\mathrm{ABG}]$ which relate their derived category to $D^{G \times \mathbb{G}_{m}}(\tilde{\mathcal{N}})$. We then use the Positivity Lemma to show that a functor constructed in [ABG] sends irreducible exotic sheaves to tilting modules over a quantum group (up to a homological shift). In section 4 we recall from $[\mathrm{AB}]$ the equivalence between $D^{G}(\tilde{\mathcal{N}})$ and a certain derived category of perverse sheaves on the affine flag variety for the Langlands dual group. It sends irreducible exotic sheaves to irreducible perverse sheaves, and the grading on Hom spaces coming from the $\mathbb{G}_{m}$-equivariant structure is related to Frobenius weights. This allows to deduce Positivity Lemma from purity of irreducible perverse sheaves (see also Remark 6 in section 2.5).

1.4. Notations. Fix an algebraically closed ground field $\mathbf{k}$ of characteristic zero.

1.4.1. Notations related to $G$. $G$ is a semi-simple algebraic group; in section 3 it will be assumed to be adjoint. The Springer map $\pi: \tilde{\mathcal{N}} \rightarrow \mathcal{N}$ was recalled above.

Let $\Lambda$ be the weight lattice of $G, \Lambda^{+} \subset \Lambda$ be the set of dominant weights and $R^{+} \subset \Lambda$ be the subsemigroup (with zero) generated by positive roots. We have the standard partial order on $\Lambda, \lambda \preceq \mu$ if $\mu-\lambda \in R^{+}$.

For $\lambda \in \Lambda^{+}$we let $V_{\lambda}$ be the corresponding irreducible representation of $G$ or of the Lie algebra $\mathfrak{g}$. We let $W_{f}$ be the Weyl group of $G$, and $W=W_{f} \ltimes \Lambda$ be the extended affine Weyl group. Let $\ell: W \rightarrow \mathbb{Z}_{\geq 0}$ be the length function. For $\lambda \in \Lambda$ let $\delta_{\lambda}$ denote the minimal length of an element $w \in W_{f}$ such that $w(\lambda) \in \Lambda^{+}$.

1.4.2. Notations for triangulated categories. For a triangulated category $\mathfrak{D}$ and $X, Y \in \mathfrak{D}$ we will use the notation $\operatorname{Hom}^{n}(X, Y)=\operatorname{Hom}(X, Y[n]), \operatorname{Hom} \bullet(X, Y)=$ $\bigoplus \operatorname{Hom}(X, Y[n])$. $n \in \mathbb{Z}$

For a set $S$ of objects in $\mathfrak{D}$ we will denote by $\ll S \gg$ the full triangulated subcategory generated by $S$; and by $\langle S\rangle$ the full subcategory generated by $S$ under extensions. Thus $\ll S \gg$ is the smallest strictly full triangulated subcategory of $\mathfrak{D}$ containing $S$; and $\langle S\rangle$ is the smallest strictly full subcategory containing $S$ and closed under extensions (we say that $\mathcal{C} \subset \mathfrak{D}$ is closed under extensions if for any $X, Y \in \mathcal{C}$ and any exact triangle $X \rightarrow Z \rightarrow Y \rightarrow X[1]$ we have $Z \in \mathfrak{D})$.

If $\mathcal{C} \subset \mathfrak{D}$ is a full triangulated subcategory we can form the quotient triangulated category $\mathfrak{D} / \mathcal{C}$; we will write $X \cong Y \bmod \mathcal{C}$ for $X, Y \in \mathfrak{D}$ meaning that the images of $X$ and $Y$ in $\mathfrak{D} / \mathcal{C}$ are isomorphic.

\footnotetext{
${ }^{2}$ The conjecture stems from discussions with D. Gaitsgory.
} 
For a category $\mathcal{C}$ let $[\mathcal{C}]$ be the set of isomorphism classes of objects in $\mathcal{C}$. If $S_{1}, S_{2}$ are subsets of [D], then $S_{1} * S_{2}$ denotes the subset of [D] consisting of classes of all objects $Z$, for which there exists an exact triangle $X \rightarrow Z \rightarrow Y \rightarrow X[1]$ with $[X] \in S_{1},[Y] \in S_{2}$.

Direct and inverse image functors on the derived categories of (quasi)coherent sheaves etc. are understood to be the corresponding derived functors, unless stated otherwise.

1.5. Acknowledgements. This is an outgrowth of the project initiated during the IAS special year in Representation Theory (1998/99), as a result of various conversations with several mathematicians, especially with M. Finkelberg and I. Mirković; I am much indebted to them. I also thank D. Gaitsgory, V. Ginzburg and V. Ostrik for inspiring communications. I thank J. Humphreys, J. Kamnitzer, I. Mirković and the referees for pointing out many typos and exposition lapses.

The author was supported by the NSF grant DMS-0071967, and was employed by the Clay Mathematical Institute at the time when ideas of this paper were being worked out; he was partially supported by DARPA grant HR0011-04-1-0031 during the final stage of his work on the paper.

\section{Coherent sheaves: exotic $t$-Structure, and Positivity Lemma.}

In this section we define the exotic $t$-structures on $D^{G}(\tilde{\mathcal{N}}), D^{G \times \mathbb{G}_{m}}(\tilde{\mathcal{N}})$. The construction will use the notion of an exceptional set in a triangulated category; we start by recalling this notion.

2.1. Exceptional sets and quasi-hereditary hearts. Most of the material in this section is borrowed from [BK] or [BGS].

2.1.1. Admissible subcategories. For a subcategory $\mathcal{C}$ in an additive category $\mathfrak{D}$ let us (following [BK]) write $\mathcal{C}^{\perp}=\left(\mathcal{C}^{\perp}\right)_{\mathfrak{D}}\left(\right.$ respectively $\left.{ }^{\perp} \mathcal{C}=\left({ }^{\perp} \mathcal{C}\right)_{\mathfrak{D}}\right)$ for the strictly full subcategory in $\mathfrak{D}$ consisting of objects $X$ for which $\operatorname{Hom}(A, X)=0$ (respectively $\operatorname{Hom}(X, A)=0)$ for all $A \in \mathcal{C}$. The subcategories $\mathcal{C}^{\perp},{ }^{\perp} \mathcal{C}$ are called respectively the right and left orthogonal of $\mathcal{C}$.

A full triangulated subcategory $\mathcal{C} \subset \mathfrak{D}$ is called right (respectively, left) admissible if the following equivalent conditions hold (see, e.g., [BK], Proposition 1.5).

a) The inclusion functor $\mathcal{C} \hookrightarrow \mathfrak{D}$ has a right (respectively, left) adjoint.

b) $\mathfrak{D}=\left\langle\mathcal{C}, \mathcal{C}^{\perp}\right\rangle$ (respectively, $\mathfrak{D}=\left\langle\mathcal{C},{ }^{\perp} \mathcal{C}\right\rangle$ ).

c) $[\mathfrak{D}]=[\mathcal{C}] *\left[\mathcal{C}^{\perp}\right]\left(\right.$ respectively, $\left.[\mathfrak{D}]=\left[{ }^{\perp} \mathcal{C}\right] *[\mathcal{C}]\right)$.

(See 1.4.2 for notations.)

If $\mathcal{C} \subset \mathfrak{D}$ is a right (respectively, left) admissible subcategory, then $\mathcal{C}^{\perp}$ (respectively, ${ }^{\perp} \mathcal{C}$ ) is left (right) admissible.

2.1.2. Exceptional sets. Let $\mathfrak{D}$ be a $\mathbf{k}$-linear triangulated category. We assume it is of finite type, i.e. the $\mathbf{k}$ vector space $\operatorname{Hom}^{\bullet}(X, Y)$ is finite dimensional for any objects $X, Y$ of $\mathfrak{D}$.

An ordered subset $\nabla=\left\{\nabla^{i}, i \in I\right\}$ of $\mathrm{Ob}(\mathfrak{D})$ is called exceptional if we have $\operatorname{Hom}^{\bullet}\left(\nabla^{i}, \nabla^{j}\right)=0$ for $i<j ; \operatorname{Hom}^{n}\left(\nabla^{i}, \nabla^{i}\right)=0$ for $n \neq 0$, and $\operatorname{End}\left(\nabla^{i}\right)=\mathbf{k}$.

Let $\nabla=\left\{\nabla^{i}, i \in I\right\}$ be an exceptional set. For $i \in I$ let $\mathfrak{D}_{<i}$ be the full triangulated subcategory generated by $\nabla_{j}, j<i$. 
Let $\Delta=\left\{\Delta_{i}, i \in I\right\}$ be another subset of $\mathrm{Ob}(\mathfrak{D})$ (in bijection with $\nabla$ ). We say that $\Delta$ is dual to $\nabla$ if

$$
\operatorname{Hom}^{\bullet}\left(\Delta_{n}, \nabla^{i}\right)=0 \text { for } n>i
$$

and there exists an isomorphism

$$
\Delta_{n} \cong \nabla^{n} \quad \bmod \mathfrak{D}_{<n}
$$

It is easy to see then that $\operatorname{Hom}^{\bullet}\left(\Delta_{i}, \nabla^{i}\right)=\mathbf{k}$, and $\operatorname{Hom}^{\bullet}\left(\Delta_{i}, \nabla^{j}\right)=0$ for $i \neq j$; that the dual set equipped with the opposite ordering is exceptional; and that it is unique if it exists (up to an isomorphism, which is fixed uniquely by fixing (2)); see, e.g., Lemma 2 in [B2]. Moreover, we have

Proposition 1. Let $\nabla \subset O b(\mathfrak{D})$ be a finite exceptional set.

a) The triangulated subcategory $\mathcal{C}=\ll \nabla \gg$ generated by $\nabla$ is both left and right admissible.

b) The dual exceptional set exists.

Proof. (a) is Corollary 2.10 in $[\mathrm{BK}]$. (b) follows from (a): set $\mathcal{C}_{n}={ }^{\perp}\left\langle\nabla^{1}, \ldots, \nabla^{n-1}\right\rangle$; then $\mathcal{C}_{n}$ is a right admissible subcategory, thus there exists a functor $\Pi_{n}: \mathfrak{D} \rightarrow \mathcal{C}_{n}$ right adjoint to the inclusion. We set $\Delta_{n}=\Pi_{n}\left(\nabla^{n}\right)$; the desired properties of $\Delta_{n}$ follow by a straightforward verification.

2.1.3. $t$-structure of an exceptional set and quasi-hereditary categories. Let $\nabla=$ $\left(\nabla^{i}\right)$ be an exceptional set in a finite type triangulated $\mathbf{k}$-linear category $\mathfrak{D}$; here $i$ runs over $\mathbb{Z}_{>0}$, or $[1, . ., n]$. Let $\Delta_{i}$ be the dual exceptional set. Assume that $\mathfrak{D}=\ll \nabla \gg$.

We refer, e.g., to [B2] for a proof of the next Proposition; here we remark only that the $t$-structure is obtained by applying gluing of $t$-structures construction from [BBD], §1.4.

Proposition 2. a) There exists a unique t-structure $\left(\mathfrak{D}^{\geq 0}, \mathfrak{D}^{<0}\right)$ on $\mathfrak{D}$, such that $\nabla^{i} \in \mathfrak{D}^{\geq 0} ; \Delta^{i} \in \mathfrak{D}^{\leq 0}$. Moreover, $\mathfrak{D}^{\geq 0}, \mathfrak{D}^{<0}$ are given by

$$
\begin{aligned}
& \mathfrak{D}^{\geq 0}=\left\langle\left\{\nabla^{i}[d], i \in I, d \leq 0\right\}\right\rangle ; \\
& \mathfrak{D}^{<0}=\left\langle\left\{\Delta_{i}[d], i \in I, d>0\right\}\right\rangle .
\end{aligned}
$$

b) The t-structure is bounded.

c) For $X \in \mathfrak{D}$ we have $X \in \mathfrak{D} \geq 0 \Longleftrightarrow \operatorname{Hom}^{<0}\left(\Delta_{i}, X\right)=0 \forall i$;

$X \in D^{<0} \Longleftrightarrow \operatorname{Hom}^{\leq 0}\left(X, \nabla^{i}\right)=0 \forall i$.

d) Let $\mathcal{A}$ denote the heart of the above t-structure. Then every object of $\mathcal{A}$ has finite length. For every $i$ the image $L_{i}$ of the canonical arrow $\tau_{\geq 0}\left(\Delta_{i}\right) \rightarrow \tau_{\leq 0}\left(\nabla^{i}\right)$ is irreducible. The objects $L_{i}$ are pairwise non-isomorphic, and every irreducible object in $\mathcal{A}$ is isomorphic to $L_{i}$ for some $i$. The $t$-structure induces a t-structure on $\mathfrak{D}_{i}=\ll\left\{\nabla_{1}, \ldots, \nabla_{i}\right\} \gg$; the heart of the latter, $\mathcal{A}_{i}$, is the Serre subcategory in $\mathcal{A}$ generated by $L_{1}, \ldots L_{i}$. The map $\tau_{\geq 0}\left(\Delta_{i}\right) \rightarrow L_{i}$ is a projective cover of $L_{i}$ in $\mathcal{A}_{i}$, and $L_{i} \rightarrow \tau_{\leq 0}\left(\nabla^{i}\right)$ is an injective hull of $L_{i}$ in $\mathcal{A}_{i}$.

Remark 1. An abelian category satisfying the properties summarized in Proposition 2 (d) is called a quasi-hereditary category (or Kazhdan-Lusztig type category, or highest weight category) see, e.g., [CPS] or [BGS]. 
Remark 2. The reader can keep in mind the following example. Let $\mathfrak{D}$ be a full subcategory in the bounded derived category of sheaves of vector spaces on a reasonable topological space (or of the "derived category" of $l$-adic etale sheaves on a reasonable scheme), consisting of complexes whose cohomology is smooth along a fixed (reasonable) stratification. Assume for simplicity that the strata $\Sigma_{i}$ are connected and simply-connected, and satisfy $H^{>0}\left(\Sigma_{j}\right)=0$; we write $j<i$ if $\Sigma_{j}$ lies in the closure of $\Sigma_{i}$. Let $\mathfrak{j}_{i}$ denote the embedding of $\Sigma_{i}$ in the space. Let $p_{i}$ be arbitrary integers. Then objects $\nabla^{i}=\mathfrak{j}_{*}\left(\overline{\mathbb{Q}_{l}}\left[p_{i}\right]\right)$ form an exceptional set generating $\mathfrak{D}$, and $\Delta_{i}=\mathfrak{j}_{!}\left(\overline{\mathbb{Q}}\left[p_{i}\right]\right)$ is the dual set. The $t$-structure of this exceptional set is the perverse $t$-structure [BBD] corresponding to perversity $p=\left(p_{i}\right)$.

2.1.4. Mutation of an exceptional set. Let $(I, \preceq)$ be an ordered set, and $\nabla^{i} \in \mathfrak{D}$, $i \in I$ be an exceptional set. Let $\leq$ be another order on $I$; we assume that either $I$ is finite, or the ordered set $(I, \leq)$ is isomorphic to $\mathbb{Z}_{>0}$. Recall that $\mathfrak{D}_{\leq i}=\ll$ $\left\{\nabla^{j} \mid j \leq i\right\} \gg ; \mathfrak{D}_{<i}=\ll\left\{\nabla^{j} \mid j<i\right\} \gg$.

Let $(I, \preceq)$ be an ordered set, and $\nabla^{i} \in \mathfrak{D}, i \in I$ be an exceptional set. Let $\leq$ be another order on $I$; we assume that either $I$ is finite, or the ordered set $(I, \leq)$ is isomorphic to $\mathbb{Z}_{>0}$. Recall that $\mathfrak{D}_{\leq i}=\ll\left\{\nabla^{j} \mid j \leq i\right\} \gg ; \mathfrak{D}_{<i}=\ll\left\{\nabla^{j} \mid j<i\right\} \gg$.

Lemma 1. a) For $i \in I$ there exists a unique (up to a unique isomorphism) object $\nabla_{\text {mut }}^{i}$ such that $\nabla_{\text {mut }}^{i} \in \mathfrak{D}_{<i} \cap \mathfrak{D}_{<i}^{\perp}$, and $\nabla_{\text {mut }}^{i} \cong \nabla^{i} \bmod \mathfrak{D}_{<i}$.

b) The objects $\nabla_{\text {mut }}^{i}$ form an exceptional set indexed by $(I, \leq)$.

c) We have $\mathfrak{D}_{\leq i}=\ll\left\{\nabla_{\text {mut }}^{j} \mid j \leq i\right\} \gg, \ll \nabla \gg=\ll \nabla_{\text {mut }} \gg$.

Proof. The proof of uniqueness in (a) is standard. Let $\Pi_{i}$ denote the projection functor $\mathfrak{D}_{<i} \rightarrow \mathfrak{D}_{<i} / \mathfrak{D}_{<i}$. Let $\Pi_{i}^{r}$ denote the right adjoint functor, it exists by Proposition 1(a). We set $\nabla_{m u t}^{i}=\Pi_{i}^{r} \circ \Pi_{i}\left(\nabla^{i}\right)$; it is immediate to check that $\nabla_{m u t}^{i}$ satisfies the requirements of part (a) and forms an exceptional set. Part (c) is then easily proved by induction.

We will say that the exceptional set $\left(\nabla_{\text {mut }}^{i}\right)$ is the $\leq$ mutation of $\left(\nabla^{i}\right)$. It is clear from the definition that it depends only on the second order $\leq$, but not on the original order $\preceq$.

Remark 3. The above notion of mutation of an exceptional set is related to the action of the braid group on the set of exceptional sets in a given triangulated category constructed in [BK] (this action is also called the action by mutations). Assume for simplicity that an exceptional set $\nabla$ is finite. Then a pair of orders $(\preceq, \leq)$ on $\nabla$ defines a permutation $\sigma$ of $\nabla$. The $(\leq)$ mutation of $\nabla$ can also be obtained by the action of the element $\tilde{\sigma}$ of the braid group on $\nabla$; here $\tilde{\sigma}$ is the minimal length lifting of $\sigma$ to an element of the braid group.

2.1.5. Graded version. Here we extend (in a rather straightforward manner) the above results about exceptional sets to some infinite exceptional sets. An example of the situation discussed in this subsection arises from a graded quasi-hereditary algebra: the bounded derived category of finitely generated modules is generated by a finite exceptional set, but the corresponding derived category of graded modules is not, because its Grothendieck group has infinite rank (the set of irreducibles has a free action of $\mathbb{Z}$ by shifts of grading, hence is infinite).

Let $\mathfrak{D}$ be a k-linear triangulated category equipped with a triangulated auto-equivalence which we call shift of grading (or just shift), and denote $\mathcal{F} \mapsto \mathcal{F}(1)$; its powers are denoted $\mathcal{F} \mapsto \mathcal{F}(n)$. For $X, Y \in \mathfrak{D}$ set 
$\operatorname{Hom}_{g r}^{i}(X, Y)=\bigoplus_{n} \operatorname{Hom}^{i}(X, Y(n))$. Assume that $\mathfrak{D}$ is of graded finite type, i.e., $\operatorname{Hom}_{g r}^{\bullet}(X, Y)$ is finite dimensional for all $X, Y \in \mathfrak{D}$.

An ordered set $\nabla^{i} \in \mathfrak{D}$ is graded exceptional if $\operatorname{Hom}_{g r}^{\bullet}\left(\nabla^{i}, \nabla^{j}\right)=0$ for $i<j$, and $\operatorname{Hom}_{g r}^{\bullet}\left(\nabla^{i}, \nabla^{i}\right)=\mathbf{k}$ for all $i$. We will (slightly abusing the terminology) say that a graded exceptional set $\nabla^{i}$ is generating if $\mathfrak{D}=\ll\left\{\nabla^{i}(k) \mid i \in I, k \in \mathbb{Z}\right\} \gg$.

We record the graded analogs of the previous two Propositions.

The dual set $\Delta_{i}$ to a graded exceptional set is defined by conditions

$$
\begin{gathered}
\operatorname{Hom}_{g r}^{\bullet}\left(\Delta_{n}, \nabla^{i}\right)=0 \text { for } n>i ; \\
\Delta_{n} \cong \nabla^{n} \bmod \mathfrak{D}_{<n}^{g r},
\end{gathered}
$$

where $\mathfrak{D}_{<n}^{g r}=\ll \nabla^{i}(k) \mid i<n, k \in \mathbb{Z} \gg$.

Again, it is easy to see that $\operatorname{Hom}_{g r}^{\bullet}\left(\Delta_{i}, \nabla^{i}\right)=\mathbf{k}$, and $\operatorname{Hom}_{g r}^{\bullet}\left(\Delta_{i}, \nabla^{j}\right)=0$ for $i \neq j$, that the dual set equipped with the opposite ordering is exceptional and that it is unique if it exists.

Proposition 3. Let $\nabla \subset O b(\mathfrak{D})$ be a finite graded exceptional set.

a) The triangulated subcategory $\mathcal{C}=\ll \nabla \gg$ generated by $\nabla(k), k \in \mathbb{Z}$ is both left and right admissible.

b) The dual graded exceptional set exists.

Proof. (b) follows from (a) as in Proposition 1. We prove (a) by induction in the number of elements in $\nabla$. If this number is one, then the functors $X \mapsto$ $\bigoplus_{n \in \mathbb{Z}} \operatorname{Hom}^{\bullet}\left(\nabla^{1}(n), X\right) \otimes \nabla^{1}(n), X \mapsto \bigoplus_{n \in \mathbb{Z}} \operatorname{Hom}^{\bullet}\left(X, \nabla^{1}(-n)\right)^{*} \otimes \nabla^{1}(n)$, from $\mathfrak{D}$ to $\ll\left\{\nabla^{1}(k), k \in \mathbb{Z}\right\} \gg$ are readily seen to be, respectively, right and left adjoint to the inclusion. This provides the base of the induction. The induction step readily follows from the next Lemma applied to $\mathcal{C}_{1}=\ll \nabla^{1}(n) \gg, \mathcal{C}_{2}=\ll \nabla^{2}(n), \ldots, \nabla^{n} \gg$.

Lemma 2. Let $\mathcal{C}_{1}, \mathcal{C}_{2}$ be full triangulated subcategories of $\mathfrak{D}$, such that $\mathcal{C}_{2} \subset \mathcal{C}_{1}^{\perp}$; define a full triangulated subcategory $\mathcal{C} \subset \mathfrak{D}$ by $\mathcal{C}=\ll \mathcal{C}_{1} \cup \mathcal{C}_{2} \gg ;$ thus $[\mathcal{C}]=\left[\mathcal{C}_{1}\right] *\left[\mathcal{C}_{2}\right]$. Assume that both $\mathcal{C}_{1}$ and $\mathcal{C}_{2}$ are left and right admissible in $\mathfrak{D}$. Then $\mathcal{C}$ is also left and right admissible in $\mathfrak{D}$.

Proof. See [BK], Proposition 1.12.

We now assume that $\mathfrak{D}=\ll \nabla(k) \gg, k \in \mathbb{Z}$ where $\nabla=\left(\nabla^{i}\right), i \in I$ is a graded exceptional set, where the ordered set $I$ is either finite or isomorphic to the ordered set of positive integers. We let $\Delta_{i}$ be the dual graded exceptional set.

The proofs of the following statements are parallel to the proof of Proposition 2 (see [B2]) and Lemma 1 respectively.

Proposition 4. a) There exists a unique t-structure $\left(\mathfrak{D}^{\geq 0}, \mathfrak{D}^{<0}\right)$ on $\mathfrak{D}$, such that $\nabla^{i}(k) \in \mathfrak{D}^{\geq 0}, \Delta^{i}(k) \in \mathfrak{D}^{\leq 0}$ for all $k \in \mathbb{Z}, i \in I$. Moreover, $\mathfrak{D}^{\geq 0}, \mathfrak{D}^{<0}$ are given by

$$
\begin{aligned}
& \mathfrak{D}^{\geq 0}=\left\langle\left\{\nabla^{i}(k)[d], i \in I, d \leq 0, k \in \mathbb{Z}\right\}\right\rangle ; \\
& \mathfrak{D}^{<0}=\left\langle\left\{\Delta_{i}(k)[d], i \in I, d>0, k \in \mathbb{Z}\right\}\right\rangle .
\end{aligned}
$$

b) The t-structure is bounded.

c) For $X \in \mathfrak{D}$ we have: $X \in \mathfrak{D}^{\geq 0} \Longleftrightarrow \operatorname{Hom}_{g r}^{<0}\left(\Delta_{i}, X\right)=0 \forall i$;

$X \in D^{<0} \Longleftrightarrow \operatorname{Hom}_{g r}^{\leq 0}\left(X, \nabla^{i}\right)=0 \forall i$.

d) Let $\mathcal{A}$ denote the heart of the above t-structure. Then every object of $\mathcal{A}$ has finite length. For every $i, k$ the image $L_{i}(k)$ of the canonical arrow $\tau_{\geq 0}\left(\Delta_{i}(k)\right) \rightarrow$ 
$\tau_{\leq 0}\left(\nabla^{i}(k)\right)$ is irreducible. The objects $L_{i}(k)$ are pairwise non-isomorphic, and every irreducible object in $\mathcal{A}$ is isomorphic to $L_{i}(k)$ for some $i, k$. The $t$-structure induces a t-structure on $\mathfrak{D}_{i}=\ll\left\{\nabla_{1}(k), \ldots, \nabla_{i}(k)\right\} \gg, k \in \mathbb{Z}$; the heart of the latter, $\mathcal{A}_{i}$, is the Serre subcategory in $\mathcal{A}$ generated by $L_{1}(k), \ldots L_{i}(k), k \in \mathbb{Z}$. The map $\tau_{\geq 0}\left(\Delta_{i}(k)\right) \rightarrow L_{i}(k)$ is a projective cover of $L_{i}(k)$ in $\mathcal{A}_{i}$, and $L_{i}(k) \rightarrow \tau_{\leq 0}\left(\nabla^{i}\right)$ is an injective hull of $L_{i}(k)$ in $\mathcal{A}_{i}$.

Lemma 3. Let $(I, \preceq)$ be an ordered set, and $\nabla^{i} \in \mathfrak{D}, i \in I$ be a graded exceptional set. Let $\leq$ be another order on $I$; we assume that either $I$ is finite, or the ordered set $(I, \leq)$ is isomorphic to $\mathbb{Z}_{>0}$. Set $\mathfrak{D}_{\leq i}=\ll\left\{\nabla^{j}(k) \mid j \leq i, k \in \mathbb{Z}\right\} \gg ; \mathfrak{D}_{<i}=\ll$ $\left\{\nabla^{j}(k) \mid j<i, k \in \mathbb{Z}\right\} \gg$.

a) For $i \in I$ there exists a unique (up to a unique isomorphism) object $\nabla_{\text {mut }}^{i}$ such that $\nabla_{\text {mut }}^{i} \in \mathfrak{D}_{\leq i} \cap \mathfrak{D}_{<i}^{\perp}$, and $\nabla_{\text {mut }}^{i} \cong \nabla^{i} \bmod \mathfrak{D}_{<i}$.

b) The objects $\nabla_{\text {mut }}^{i}$ form a graded exceptional set indexed by $(I, \leq)$.

c) We have $\mathfrak{D}_{\leq i}=\ll\left\{\nabla_{\text {mut }}^{j}(k) \mid j \leq i, k \in \mathbb{Z}\right\} \gg, \ll \nabla \gg=\ll \nabla_{\text {mut }} \gg$.

Thus the notion of a mutation of a graded exceptional set is defined.

2.1.6. Tilting objects. Let $\mathfrak{D}$ be a triangulated category generated by an exceptional set $\nabla$, let $\Delta$ be the dual set, and let $\mathcal{A}$ be the heart of the corresponding $t$-structure.

Recall that an object $X \in \mathcal{A}$ is called tilting if it has a filtration with associated graded $N^{i}=\tau_{\leq 0}\left(\nabla^{i}\right)$ and also has a filtration with associated graded $M_{i}=\tau_{\geq 0}\left(\Delta_{i}\right)$.

Classification of indecomposable tilting objects follows from $[\mathrm{R}]$ (cf. also $[\mathrm{BBM}]$ ): for every $i \in I$ there exists a unique (up to an isomorphism) indecomposable tilting object $T_{i} \in \mathcal{A}$ which lies in $\mathcal{A}_{\leq i}$ but not in $\mathcal{A}_{<i}$; every indecomposable tilting object is isomorphic to $T_{i}$ for some $i \in I$. We have a surjective morphism $T_{i} \rightarrow \nabla^{i}$ whose kernel has a filtration with associated graded $\nabla^{j}, j<i$; and an injective morphism $\Delta_{i} \rightarrow T_{i}$ whose cokernel has a filtration with associated graded $\Delta_{j}, j<i$.

Lemma 4. Assume that $\operatorname{Hom}^{<0}\left(\nabla^{i}, \nabla^{j}\right)=0=\operatorname{Hom}^{<0}\left(\Delta_{i}, \Delta_{j}\right)$.

Let $X \in \mathfrak{D}$ be an object, such that $\operatorname{Hom}^{>0}\left(\Delta_{k}, X\right)=0=\operatorname{Hom}^{>0}\left(X, \nabla_{k}\right)$ for all $k$. Then $X$ lies in $\mathcal{A}$ and it is a tilting object therein.

Proof. The first condition shows that $\Delta_{i}, \nabla^{i} \in \mathcal{A}$ for all $i$; thus $M_{i}=\tau_{\geq 0}\left(\Delta_{i}\right)=$ $\Delta_{i}$ and $N^{i}=\tau_{\leq 0}\left(\nabla^{i}\right)=\nabla^{i}$.

The second one implies by a standard argument that $X \in\left\langle\nabla_{i}[n]\right\rangle \cap\left\langle\Delta_{i}[-n]\right\rangle$, $i \in I, n \in \mathbb{Z}_{\geq 0}$. In particular, $X \in \mathfrak{D}^{\leq 0} \cap \mathfrak{D}^{\geq 0}=\mathcal{A}$; thus $\operatorname{Hom}^{i}\left(\Delta_{k}, X\right)=0=$ $\operatorname{Hom}^{i}\left(X, \nabla_{k}\right)$ for $i \neq 0$. It is well known that the latter condition implies that $X \in\left\langle\Delta_{i}\right\rangle \cap\left\langle\nabla^{i}\right\rangle$; since $M_{i}=\Delta_{i}, N^{i}=\nabla^{i}$ we see that $X$ is tilting.

Remark 4. Notice that we have deduced vanishing of $\operatorname{Hom}^{i}\left(\Delta_{k}, X\right), \operatorname{Hom}^{i}\left(X, \nabla_{k}\right)$ for $i<0$ from the corresponding vanishing for $i>0$. This elementary argument acquires interesting consequences when it is taken together with a derived Koszul equivalence (see Introduction, discussion before the Conjecture in §1.2). This is illustrated by the methods of the present paper. Namely, we will see later that for a certain class of objects $X \in D^{b}\left(\mathrm{U}_{q}-\bmod ^{0}\right)$, the vanishing of $\operatorname{Hom}^{i}\left(\Delta_{k}, X\right)$, $\operatorname{Hom}^{i}\left(X, \nabla_{k}\right)$ for $i>0$ follows from purity of some irreducible perverse sheaves on the affine flag variety; while the corresponding vanishing for $i \neq 0$ can be interpreted as pointwise purity of these irreducible perverse sheaves.

Recall that purity is a general property of irreducible perverse $l$-adic sheaves proved in $[\mathrm{BBD}]$, while pointwise purity does not hold for a general IC sheaf, but is 
known to hold, e.g., for IC sheaves of (affine) Schubert varieties. However, Lemma 4 can be used to show that pointwise purity of irreducible objects in a category $\mathcal{A}$ of perverse sheaves formally follows from their purity, provided that a derived Koszul equivalence between $D^{b}(\mathcal{A})$ and some other (reasonable) derived category is given. This is an illustration of the relation ${ }^{3}$ between the pointwise purity property and the Koszul duality formalism.

2.2. Sheaves on the nilpotent cone. In this subsection we recall some results and notations from [B2].

Till the end of this section $2 G$ is assumed to be an arbitrary semi-simple algebraic group.

For $\lambda \in \Lambda$ we let $\mathcal{O}_{G / B}(\lambda) \in \operatorname{Coh}^{G}(G / B)$ be the corresponding line bundle on $G / B$, and we set $\mathcal{O}_{\lambda}=\operatorname{pr}^{*}\left(\mathcal{O}_{G / B}(\lambda)\right) \in \operatorname{Coh}^{G}(\tilde{\mathcal{N}})$, where $p r: \tilde{\mathcal{N}} \rightarrow G / B$ is the projection.

Recall that we consider the action of the multiplicative group $\mathbb{G}_{m}$ on $\tilde{\mathcal{N}}$ given by $t:(\mathfrak{b}, x) \mapsto\left(\mathfrak{b}, t^{2} x\right)$. Let us equip $\mathcal{O}_{G / B}(\lambda)$ with the trivial $\mathbb{G}_{m}$ action, thus we get an object $\mathcal{O}_{G / B}(\lambda, 0) \in C o h^{G \times \mathbb{G}_{m}}(G / B)$ (where $\mathbb{G}_{m}$ acts trivially on $G / B$ ); and then set $\mathcal{O}_{\lambda, 0}=\operatorname{pr}^{*}\left(\mathcal{O}_{G / B}(\lambda, 0)\right) \in \operatorname{Coh}^{G \times \mathbb{G}_{m}}(\tilde{\mathcal{N}})$.

The perverse coherent $t$-structure on $D^{G}(\mathcal{N})$ of the middle perversity ${ }^{4}$ is defined by: $\mathcal{F} \in D^{p, \leq 0}(\mathcal{N}) \Longleftrightarrow i_{O}^{*}(\mathcal{F}) \in D^{\leq d^{-} d_{O}}\left(\operatorname{Coh}^{G}(O)\right), \mathcal{F} \in D^{p,>0}(\mathcal{N}) \Longleftrightarrow$ $i_{O}^{!}(\mathcal{F}) \in D^{>d-d_{O}}\left(\operatorname{Coh}^{G}(O)\right)$, where $O$ runs over the set of $G$ orbits in $\mathcal{N}$, and $d_{O}=\frac{\operatorname{dim}(O)}{2}, d=\frac{\operatorname{dim} \mathcal{N}}{2}$. All objects in the heart of this $t$-structure have finite length. As was pointed out in the Introduction, for a pair $(O, \mathcal{L})$, where $O \subset \mathcal{N}$ is a $G$ orbit and $\mathcal{L}$ is an irreducible $G$-equivariant vector bundle on $O$, there is a unique irreducible object $I C_{O, \mathcal{L}}$ in the heart supported on the closure of $O$ and satisfying $\left.I C_{O, \mathcal{L}}\right|_{O}=\mathcal{L}\left[d_{O}-d\right]$.

We will use the bijection (constructed in [B2]) between $\Lambda^{+}$and the set of pairs $(O, \mathcal{L})$ as above; we denote it by $\lambda \leftrightarrow\left(O_{\lambda}, \mathcal{L}_{\lambda}\right)$. For $\lambda \in \Lambda$ we set $A_{\lambda}=\pi_{*}\left(\mathcal{O}_{\lambda}\right)$ (recall that $\pi_{*}$ denotes the derived direct image functor).

Set $D_{\preceq \lambda}^{G}(\mathcal{N})=\ll\left\{A_{\nu} \mid \nu \in \Lambda^{+}, \nu \preceq \lambda\right\} \gg, D_{\prec \lambda}^{G}=\ll\left\{A_{\nu} \mid \nu \in \Lambda^{+}, \nu \prec \lambda\right\} \gg$. The following facts were proved in [B2]:

$$
\begin{gathered}
D^{G}(\mathcal{N})=\bigcup_{\lambda} D_{\preceq \lambda}^{G}(\mathcal{N}) ; \\
D^{p,>0}(\mathcal{N})=\left\langle A_{\lambda}[n] \mid \lambda \in \Lambda^{+}, n<0\right\rangle \\
D^{p, \leq 0}(\mathcal{N})=\left\langle A_{w_{o}(\lambda)}[n] \mid \lambda \in \Lambda^{+}, n \geq 0\right\rangle
\end{gathered}
$$

$$
\operatorname{dim} \operatorname{Hom}\left(A_{w_{o}(\lambda)}, A_{\lambda}\right)=1, \text { and } \operatorname{IC}_{O_{\lambda}, \mathcal{L}_{\lambda}} \cong \operatorname{Im}\left(A_{w_{o}(\lambda)} \stackrel{c_{\lambda}}{\longrightarrow} A_{\lambda}\right) \text { for } \lambda \in \Lambda^{+},
$$

where $c_{\lambda}$ is the unique (up to scaling) non-zero morphism, $I m$ denotes the image with respect to the perverse $t$-structure and $w_{o} \in W_{f}$ is the longest element;

$$
A_{\lambda} \in D_{\prec \lambda}^{G}(\mathcal{N})^{\perp} ; \quad A_{w_{o}(\lambda)} \in^{\perp} D_{\prec \lambda}^{G}(\mathcal{N}) \quad \text { for } \lambda \in \Lambda^{+}
$$

\footnotetext{
${ }^{3}$ Pointed out to me by Victor Ginzburg, cf. [BGS].

${ }^{4}$ To simplify notation we use here a normalization different from the one used in [B2]: the two $t$-structures differ by the shift by $d=\frac{\operatorname{dim} \mathcal{N}}{2}$.
} 


$$
\begin{aligned}
& A_{\lambda} \cong A_{w(\lambda)} \quad \bmod D_{\prec \lambda}^{G}(\mathcal{N}) \quad \text { for } \quad \lambda \in \Lambda^{+}, w \in W_{f} \\
& D_{\prec \lambda}^{G}(\mathcal{N})^{\perp}=\ll\left\{V_{\mu} \otimes \mathcal{O}_{\mathcal{N}} \mid \mu \prec \lambda\right\} \gg^{\perp} \\
& { }^{\perp}\left(D_{\prec \lambda}^{G}(\mathcal{N})\right)={ }^{\perp} \ll\left\{V_{\mu} \otimes \mathcal{O}_{\mathcal{N}} \mid \mu \prec \lambda\right\} \gg .
\end{aligned}
$$

Finally, we recall a result of $[\mathrm{B}]$, which will be used to deduce Corollary 3 in section 3.2 (Humphreys' conjecture) (and will not be used elsewhere in the paper).

Proposition 5. Let $\lambda \in \Lambda^{+} \subset W$, and let $\underline{c} \subset W$ be the two-sided cell containing $\lambda$. Let $\left(O_{\lambda}, \mathcal{L}_{\lambda}\right)$ be the orbit and the irreducible vector bundle on it, corresponding to $\lambda$ as above. Then $O_{\lambda} \subset \mathcal{N}$ is the orbit corresponding to $\underline{c}$ under the bijection defined in $[\mathrm{Lc}]$.

2.3. Exotic $t$-structure. Some of the arguments appearing in this section can be replaced by shorter ones relying on results of $[\mathrm{AB}]$ or $[\mathrm{ABG}]$. We found it worthwhile, however, to present direct proofs; though the intuition comes from $[\mathrm{AB}]$, the techniques employed in this section go back at least to [Dem].

The category $D^{G \times \mathbb{G}_{m}}(\tilde{\mathcal{N}})$ is equipped with a shift functor $\mathcal{F} \mapsto \mathcal{F}(1)$, where $\mathcal{F}(1)$ stands for the tensor product of $\mathcal{F}$ with the tautological character of $\mathbb{G}_{m}$. For $\mathcal{F}, \mathcal{G} \in D^{G \times \mathbb{G}_{m}}(\tilde{\mathcal{N}})$ we have $\operatorname{Hom}_{D^{G}(\tilde{\mathcal{N}})}^{\bullet}(\mathcal{F}, \mathcal{G})=\bigoplus_{n} \operatorname{Hom}_{D^{G \times G_{m}}(\tilde{\mathcal{N}})}^{\bullet}(\mathcal{F}, \mathcal{G}(n))$, where the restriction of equivariance functor is omitted from the notation.

Set $\mathcal{O}_{\lambda, n}=\mathcal{O}_{\lambda, 0}(n)$.

Lemma 5. We have $\operatorname{Hom}_{D^{G}(\tilde{\mathcal{N}})}^{\bullet}\left(\mathcal{O}_{\lambda}, \mathcal{O}_{\mu}\right)=0$ unless $\lambda \succeq \mu$. Also, $\operatorname{Hom}_{D^{G}(\tilde{\mathcal{N}})}^{\bullet}\left(\mathcal{O}_{\lambda}, \mathcal{O}_{\lambda}\right)=\mathbf{k}$.

Proof. We have to check that $R^{\bullet} \Gamma^{G}\left(\mathcal{O}_{\nu}\right)=0$ if $-\nu \notin R^{+}$, and $R^{\bullet} \Gamma^{G}(\mathcal{O})=$ k. Here by $R \Gamma^{G}$ we mean the derived functor of the functor $\mathcal{F} \mapsto \Gamma(\mathcal{F})^{G}=$ $\operatorname{Hom}_{C o h^{G}}(\mathcal{O}, \mathcal{F})$. We have $R^{i} \Gamma^{G}(\mathcal{F})=\left(R^{i}(\mathcal{F})\right)^{G}$, see, e.g., [B1].

We first prove that for $\mathcal{F} \in D^{G}(\tilde{\mathcal{N}})$ there is a canonical isomorphism

$$
R^{i} \Gamma^{G}(\mathcal{F}) \cong\left(H^{i}\left(\mathfrak{n},\left.\mathcal{F}\right|_{\mathfrak{n}}\right)\right)^{T}
$$

Recalling that $\tilde{\mathcal{N}}=G \times{ }_{B} \mathfrak{n}$, we see that $(11)$ holds when $i=0$ and $\mathcal{F} \in \operatorname{Coh}^{G}(\tilde{\mathcal{N}})$. It remains to check that the $\delta$-functor $\mathcal{F} \mapsto\left(H^{i}\left(\mathfrak{n},\left.\mathcal{F}\right|_{\mathfrak{n}}\right)\right)^{T}$ is effaceable. The category $Q \operatorname{Coh}^{G}(\tilde{\mathcal{N}})$ of $G$-equivariant quasi-coherent sheaves has enough objects adjusted to $\Gamma$ (and hence to $\Gamma^{G}$ ), which are of the form $A v(\mathcal{F}), \mathcal{F} \in Q \operatorname{Coh}(\tilde{\mathcal{N}})$; here $A v$ : $Q \operatorname{Coh}(\tilde{\mathcal{N}}) \rightarrow Q \operatorname{Coh}^{G}(\tilde{\mathcal{N}})$ is the "averaging" functor, right adjoint to the forgetful functor $Q \operatorname{Coh}^{G}(\tilde{\mathcal{N}}) \rightarrow Q \operatorname{Coh}(\tilde{\mathcal{N}})$. We have $A v(\mathcal{F})=a_{*} \operatorname{pr}^{*}(\mathcal{F})$, where $p r: G \times$ $\tilde{\mathcal{N}} \rightarrow \tilde{\mathcal{N}}$, and $a: G \times \tilde{\mathcal{N}} \rightarrow \tilde{\mathcal{N}}$ are the projection and the action maps. It is easy to see that $\left.\Gamma(A v(\mathcal{F}))\right|_{\mathfrak{n}}$ is an injective module over the Lie algebra $\mathfrak{n}$. This implies (11).

We now claim that the right hand side of (11) vanishes for $\mathcal{F}=\mathcal{O}_{\lambda}$ if $-\lambda \notin$ $R^{+}$. Indeed, the standard complex for computation of $H^{\bullet}\left(\mathfrak{n},\left.\Gamma(\mathcal{O}(\lambda))\right|_{\mathfrak{n}}\right.$ is $\Lambda\left(\mathfrak{n}^{*}\right) \otimes$ $\operatorname{Sym}\left(\mathfrak{n}^{*}\right)$ with some differential; the action of $T$ is the natural action twisted by $\lambda$. If $-\lambda \notin R^{+}$, then the space of $T$ invariants in the complex vanishes, hence so does the space of $T$ invariants in its cohomology.

Proposition 6. Equip $\Lambda$ with any order compatible with the partial order $\preceq$. Then the set of objects $\mathcal{O}_{\lambda}$ indexed by $\Lambda$ with this order is an exceptional set generating $D^{G}(\tilde{\mathcal{N}})$. 
The set $\mathcal{O}_{\lambda, 0}$ is a graded exceptional set in $D^{G \times \mathbb{G}_{m}}(\tilde{\mathcal{N}})$, and $D^{G \times \mathbb{G}_{m}}(\tilde{\mathcal{N}})=\ll$ $\mathcal{O}_{\lambda, n} \gg, \lambda \in \Lambda, n \in \mathbb{Z}$.

Proof. The set in question is (graded) exceptional by Lemma 5 . The set $\mathcal{O}_{\lambda}$ generates $D^{G}(\tilde{\mathcal{N}})$ by [B2], Corollary 2 on p.13; $\mathcal{O}_{\lambda, n}$ generate $D^{G \times \mathbb{G}_{m}}(\tilde{\mathcal{N}})$ by a similar argument.

We now introduce another partial ordering $\leq$ on $\Lambda$. To this end, recall the $(2-$ sided) Bruhat partial order on the affine Weyl group $W$. For $\lambda \in \Lambda$ let $w_{\lambda}$ be the minimal length representative of the coset $W_{f} \lambda \subset W$.

We set $\mu \leq \lambda$ if $w_{\mu}$ precedes $w_{\lambda}$ in the Bruhat order.

We will use the following well-known properties of the Bruhat order:

- The two orders $\preceq, \leq$ coincide on $\Lambda^{+}$.

- The two orders $\preceq, \leq$ coincide on any $W_{f}$ orbit in $\Lambda$.

- If $\lambda \leq \mu$, then $\lambda \in \operatorname{conv}(\mu)$, where $\operatorname{conv}(\mu)$ denotes the convex hull of the $W_{f}$ orbit of $\mu$ intersected with $\Lambda$.

We fix a complete order $\leq_{\text {compl }}$ on $\Lambda$ compatible with $\leq$.

In view of the above properties of the Bruhat order we can (and will) assume that $\leq_{\text {compl }}$ satisfies the following two requirements. ${ }^{5}$

- $\left(\Lambda, \leq_{\text {compl }}\right)$ is isomorphic to $\mathbb{Z}_{\geq 0}$.

- Let $\operatorname{conv}^{0}(\mu)$ denote the complement to the $W_{f}$ orbit of $\mu$ in $\operatorname{conv}(\mu)$. Then we have

$$
\lambda \in \operatorname{conv}^{0}(\mu) \Rightarrow \lambda \leq_{\text {compl }} \mu .
$$

Proposition 6 implies that the set $\mathcal{O}_{\lambda}$ (respectively, $\mathcal{O}_{\lambda, 0}$ ) equipped with any complete order compatible with $\preceq$ is a (graded) exceptional generating set. We define the (graded) exceptional set $\nabla_{\lambda}$ (respectively, $\nabla_{\lambda, 0}$ ) to be the $\leq_{\text {compl }}$ mutation of the set $\mathcal{O}_{\lambda}$ (respectively, $\mathcal{O}_{\lambda, 0}$ ). We let $\Delta_{\lambda}$ (respectively, $\Delta_{\lambda, 0}$ ) be the dual (graded) exceptional sets. It is clear from the definitions that the forgetful functor $D^{G \times \mathbb{G}_{m}}(\tilde{\mathcal{N}}) \rightarrow D^{G}(\tilde{\mathcal{N}})$ sends $\nabla^{\lambda, 0}$ to $\nabla^{\lambda}$ and $\Delta_{\lambda, 0}$ to $\Delta_{\lambda}$

Our next goal is to get a more explicit description of $\nabla^{\lambda}, \nabla^{\lambda, 0}$. It is provided by the next Proposition 7 ; to state it we need some notations.

For a subset $S \subset \Lambda$ set $D_{S}^{G}(\tilde{\mathcal{N}})=\ll \mathcal{O}_{\lambda}\left|\lambda \in S \gg, D_{S}^{G \times \mathbb{G}_{m}}(\tilde{\mathcal{N}})=\ll \mathcal{O}_{\lambda, n}\right| \lambda \in$ $S, n \in \mathbb{Z} \gg$.

Let $\Pi_{\lambda}^{l}: D^{G}(\tilde{\mathcal{N}}) \rightarrow D_{\operatorname{conv}^{0}(\lambda)}^{G}(\tilde{\mathcal{N}})^{\perp},\left(\Pi_{\lambda}^{l}\right)^{g r}: D^{G \times \mathbb{G}_{m}}(\tilde{\mathcal{N}}) \rightarrow D_{\operatorname{conv}^{0}(\lambda)}^{G \times \mathbb{G}_{m}}(\tilde{\mathcal{N}})^{\perp}$ be the functors left adjoint to the embedding functors.

For a simple root $\alpha$ let $s_{\alpha} \in W_{f}$ be the corresponding simple reflection. Let $\pi_{\alpha}$ : $G / B \rightarrow G / P_{\alpha}$ be the projection, where $P_{\alpha}$ is the minimal parabolic corresponding to $\alpha$. Set $\tilde{\mathcal{N}}_{\alpha}=T^{*}\left(G / P_{\alpha}\right) \times_{G / P_{\alpha}} G / B$; the differential of $\pi_{\alpha}$ provides a closed embedding $i_{\alpha}: \tilde{\mathcal{N}}_{\alpha} \hookrightarrow \tilde{\mathcal{N}}$. We let $\widetilde{\pi}_{\alpha}$ denote the projection $\tilde{\mathcal{N}}_{\alpha} \rightarrow T^{*}\left(G / P_{\alpha}\right)$.

Define a functor $F_{\alpha}: D^{G}(\tilde{\mathcal{N}}) \rightarrow D^{G}(\tilde{\mathcal{N}})$ by

$$
F_{\alpha}: \mathcal{F} \mapsto\left(i_{\alpha *} \circ \tilde{\pi}_{\alpha}^{*} \circ \tilde{\pi}_{\alpha *} \circ i_{\alpha}^{!}(\mathcal{F}(\rho))\right)(-\rho) ;
$$

a functor $F_{\alpha}^{g r}: D^{G \times \mathbb{G}_{m}}(\tilde{\mathcal{N}}) \rightarrow D^{G \times \mathbb{G}_{m}}(\tilde{\mathcal{N}})$ is defined by the same formula. ${ }^{6}$

\footnotetext{
${ }^{5}$ One can use the comparison with geometry of affine flags, see section 4 , to show that the second requirement is, in fact, redundant. We still found it convenient to impose it for the sake of a technical simplification.

${ }^{6}$ The formula defines a functor on the equivariant derived category only if $\mathcal{O}_{\rho}$ carries a $G$ equivariant structure; this is true if $G$ is simply connected, but not in general. However, the
} 
Notice that each of the functors $F_{\alpha}, F_{\alpha}^{g r}$ is defined as a composition of six functors which can be grouped into three adjoint pairs: $\left(\widetilde{\pi}_{\alpha}^{*}, \widetilde{\pi}_{\alpha *}\right),\left(i_{\alpha}^{*}, i_{\alpha}^{!}\right),\left(T_{-\rho}, T_{\rho}\right)$, where we let $T_{\lambda}$ denote the functor of twist by $\mathcal{O}_{\lambda}$ (respectively, by $\mathcal{O}_{\lambda, 0}$ ).

We also define the functors $F_{\alpha}^{\prime},\left(F_{\alpha}^{\prime}\right)^{g r}$ by $\mathcal{F} \mapsto\left(i_{\alpha *} \circ \widetilde{\pi}_{\alpha}^{!} \circ \widetilde{\pi}_{\alpha *} \circ i_{\alpha}^{*}(\mathcal{F}(-\rho))\right)(\rho)$.

Thus $F_{\alpha}$ (respectively, $F_{\alpha}^{g r}$ ) is the composition of $\widetilde{\pi}_{\alpha *} \circ i_{\alpha}^{!} \circ T_{\rho}$ with its left adjoint. In particular, we get a canonical arrow $F_{\alpha} \stackrel{c a n_{\alpha}}{\longrightarrow} i d$ and similarly for $F_{\alpha}^{g r}$.

We have a canonical arrow $i d \stackrel{c a n_{\alpha}^{\prime}}{\longrightarrow} F_{\alpha}^{\prime}$ and its graded version.

Proposition 7. a) We have $\nabla^{\lambda} \cong \Pi_{\lambda}^{l}\left(\mathcal{O}_{\lambda}\right), \nabla^{\lambda, n} \cong\left(\Pi_{\lambda}^{l}\right)^{g r}\left(\mathcal{O}_{\lambda, n}\right)$. In particular, $\nabla^{\lambda}, \nabla^{\lambda, n}$ do not depend on the choice of $\leq_{\text {compl }}$.

b) Assume that $s_{\alpha}(\lambda) \preceq \lambda$. Then we have canonical distinguished triangles:

$$
\begin{aligned}
& F_{\alpha}^{g r}\left(\nabla^{\lambda, 0}\right) \stackrel{\operatorname{can}_{\alpha}}{\longrightarrow} \nabla^{\lambda, 0} \rightarrow \nabla^{s_{\alpha}(\lambda), 2}, \\
& \nabla^{\lambda, 0} \rightarrow \nabla^{s_{\alpha}(\lambda), 2} \stackrel{\operatorname{can}_{\alpha}^{\prime}}{\longrightarrow}\left(F_{\alpha}^{\prime}\right)^{g r}\left(\nabla^{s_{\alpha}(\lambda), 2}\right)
\end{aligned}
$$

and similarly with $F_{\alpha}^{g r},\left(F_{\alpha}^{\prime}\right)^{g r}, \nabla^{\lambda, 0}, \nabla^{s_{\alpha}(\lambda), 2}$ replaced by $F_{\alpha}, F_{\alpha}^{\prime}, \nabla^{\lambda}, \nabla^{s_{\alpha}(\lambda)}$.

The proof of the Proposition is preceded by two Lemmas.

Lemma 6. a) The left adjoint to $F_{\alpha}, F_{\alpha}^{g r}$ is isomorphic to $F_{\alpha}[-2], F_{\alpha}^{g r}[-2](-2)$ respectively.

b) We have $F_{\alpha} \cong F_{\alpha}^{\prime}[2], F_{\alpha}^{g r} \cong\left(F_{\alpha}^{\prime}\right)^{g r}[2](-2)$.

Proof. We use the well-known isomorphisms of $G \times \mathbb{G}_{m}$ equivariant line bundles on $\tilde{\mathcal{N}}$ :

$$
\begin{aligned}
& \mathcal{O}\left(-\tilde{\mathcal{N}}_{\alpha}\right) \cong \mathcal{O}_{\alpha,-2}, \\
& K_{G / B} \otimes \pi_{\alpha}^{*}\left(K_{G / P_{\alpha}}^{-1}\right) \cong \mathcal{O}_{-\alpha},
\end{aligned}
$$

where $K$ stands for the canonical line bundle of top degree forms. They imply canonical isomorphism:

$$
\begin{gathered}
i_{\alpha}^{!}(\mathcal{F}) \cong i_{\alpha}^{*}\left(\mathcal{F} \otimes \mathcal{O}\left(\tilde{\mathcal{N}}_{\alpha}\right)\right)[-1]=i_{\alpha}^{*}(\mathcal{F}(-\alpha, 2))[-1] \\
\tilde{\pi}_{\alpha}^{!}(\mathcal{F}) \cong \tilde{\pi}_{\alpha}^{*}(\mathcal{F}) \otimes \mathcal{O}_{G / B}\left(K_{G / B} \otimes \pi_{\alpha}^{*}\left(K_{G / P_{\alpha}}^{-1}\right)\right)[1] \cong \tilde{\pi}_{\alpha}^{*}(\mathcal{F})(-\alpha, 0)[1] .
\end{gathered}
$$

Since $\widetilde{\pi}_{\alpha}^{*}, i_{\alpha}^{*}$ are left adjoint to $\widetilde{\pi}_{\alpha *}, i_{\alpha}^{*}$ respectively, while $\widetilde{\pi}_{\alpha}^{!}, i_{\alpha}^{!}$are right adjoint to $\widetilde{\pi}_{\alpha *}, i_{\alpha}^{*}$ respectively, we get (a) by plugging in the latter isomorphisms. They also imply that

$$
F_{\alpha}^{g r} \cong T_{-2 \rho+\alpha, 0}\left(F_{\alpha}^{\prime}\right)^{g r} T_{2 \rho-\alpha, 2}
$$

Since $\langle\check{\alpha}, 2 \rho-\alpha\rangle=0$ (where $\check{\alpha}$ is the dual coroot), we see that $\mathcal{O}_{2 \rho-\alpha}$ is lifted from $G / P_{\alpha}$, hence $T_{2 \rho-\alpha, 0}$ commutes with $\left(F_{\alpha}^{\prime}\right)^{g r}$. The isomorphism in (b) follows.

Lemma 7. a) If $s_{\alpha}(\lambda) \preceq \lambda$, then we have canonical isomorphisms in (the quotient category of) $D^{G \times \mathbb{G}_{m}}(\tilde{\mathcal{N}})$ :

$$
\begin{aligned}
& \mathcal{O}_{s_{\alpha}(\lambda), 2} \cong \operatorname{cone}\left(F_{\alpha}\left(\mathcal{O}_{\lambda, 0}\right) \stackrel{\operatorname{can}_{\alpha}}{\longrightarrow} \mathcal{O}_{\lambda, 0}\right) \quad \bmod D_{\operatorname{conv}^{0}(\lambda)}^{G \times \mathbb{G}_{m}}(\tilde{\mathcal{N}}), \\
& \left.\mathcal{O}_{\lambda, 0}[1] \cong \operatorname{cone}\left(\mathcal{O}_{s(\lambda), 2}\right) \stackrel{\operatorname{can}_{\alpha}^{\prime}}{\longrightarrow} F_{\alpha}^{\prime}\left(\mathcal{O}_{s_{\alpha}(\lambda), 2}\right)\right) \quad \bmod D_{\operatorname{conv}^{0}(\lambda)}^{G \times \mathbb{G}_{m}}(\tilde{\mathcal{N}})
\end{aligned}
$$

derived category $D^{G}(\tilde{\mathcal{N}}), D^{G \times \mathbb{G}_{m}}$ is a direct summand in $D^{G_{s c}}(\tilde{\mathcal{N}}), D^{G_{s c} \times \mathbb{G}_{m}}(\tilde{\mathcal{N}})$, where $G_{s c}$ is the universal cover of $G$. It is easy to see that the functors $F_{\alpha}, F_{\alpha}^{g r}$ defined by the above formula for $G_{s c}$ preserve this direct summand, thus the functors are defined for a general $G$. This observation applies also to $F_{\alpha}^{\prime},\left(F_{\alpha}^{\prime}\right)^{g r}$. 
and also similar isomorphisms in (the quotient category of) $D^{G}(\tilde{\mathcal{N}})$.

b) The functors $F_{\alpha}, F_{\alpha}^{g r}$ preserve $D_{\operatorname{conv}(\lambda)}^{G}(\tilde{\mathcal{N}}), D_{\operatorname{conv}(\lambda)}^{G \times \mathbb{G}_{m}}(\tilde{\mathcal{N}})$.

Proof. We prove (a) and (b) together by induction. We assume that (b) is known for $\lambda \in \operatorname{conv}^{0}(\mu)$, and deduce that (a) holds for $\lambda \in W(\mu)$. Then the isomorphisms of (a) together with Lemma 6(b) show that (b) also holds for $\lambda \in W(\mu)$.

If $\langle\check{\alpha}, \lambda\rangle=0$, then $i_{\alpha}^{!}\left(\mathcal{O}_{\lambda+\rho}\right)=i_{\alpha}^{*}\left(\mathcal{O}_{\lambda+\rho-\alpha}\right)[-1]$ is isomorphic to the line bundle $\mathcal{O}_{\mathbb{P}^{1}}(-1)$ when restricted to a fiber of $\tilde{\pi}_{\alpha}$. Thus in this case we have $F_{\alpha}^{g r}\left(\mathcal{O}_{\lambda, 0}\right)=0$, so we get the desired isomorphism. Assume now that $\langle\check{\alpha}, \lambda\rangle>0$.

For a vector bundle $\mathcal{V}$ on $\tilde{\mathcal{N}}$ we have, in view of (13), a canonical (in particular, respecting $G$ or $G \times \mathbb{G}_{m}$ equivariance) short exact sequence

$$
0 \rightarrow \mathcal{V}(\alpha,-2) \rightarrow \mathcal{V} \rightarrow i_{\alpha *} i_{\alpha}^{*} \mathcal{V} \rightarrow 0
$$

Applying Serre-Grothendieck duality (and plugging in $\mathcal{V}^{*}$ for $\mathcal{V}$ ) we get a distinguished triangle

$$
i_{\alpha *} i_{\alpha}^{!} \mathcal{V} \rightarrow \mathcal{V} \rightarrow \mathcal{V}(-\alpha, 2)
$$

If a vector bundle $\mathcal{W}$ on $\tilde{\mathcal{N}}_{\alpha}$ is trivial on the fibers of the projection $\tilde{\pi}_{\alpha}$ (if $\mathcal{W}$ is an equivariant bundle, the trivialization is not required to be compatible with the equivariant structure), then we have $\tilde{\pi}_{\alpha}^{*} \widetilde{\pi}_{\alpha *} \mathcal{W} \longrightarrow \mathcal{W}$. Thus if $\mathcal{V}$ is a $G \times \mathbb{G}_{m}$ equivariant vector bundle, such that $i_{\alpha}^{!}(\mathcal{V}(\rho))[1]=i_{\alpha}^{*}(\mathcal{V}(\rho-\alpha))$ is trivial on the fibers of $\tilde{\pi}_{\alpha}$, then we have a distinguished triangle $i_{\alpha *} \circ \tilde{\pi}_{\alpha}^{*} \circ \tilde{\pi}_{\alpha *} \circ i_{\alpha}^{!}(\mathcal{V}) \rightarrow \mathcal{V} \rightarrow$ $\mathcal{V}(-\alpha, 2)$. If $i_{\alpha}^{*} \mathcal{V}(-\alpha+\rho)$ is fiberwise trivial, then we get a distinguished triangle $F_{\alpha}(\mathcal{V}) \rightarrow \mathcal{V} \rightarrow \mathcal{V}(-\alpha, 2)$.

For $\mu \in \Lambda,\langle\check{\alpha}, \mu\rangle \geq 0$, let $\mathcal{V}_{\mu, n}$ denote the pull-back under the projection $\tilde{\mathcal{N}} \rightarrow$ $G / B$ of $\pi_{\alpha}^{*} \pi_{\alpha *}\left(\mathcal{O}_{\mu, n}\right)$. It is clear that $\left.\pi_{\alpha}^{*} \pi_{\alpha *}\left(\mathcal{O}_{\mu}\right)\right)$ is trivial on the fibers of $\pi_{\alpha}$. It is also easy to see that this vector bundle carries a filtration with associated graded $\mathcal{O}_{\mu, n}, \mathcal{O}_{\mu-\alpha, n}, \ldots, \mathcal{O}_{s_{\alpha}(\mu), n}$

Set $\mathcal{V}=\mathcal{V}_{\lambda-\rho, 0}(\rho, 0)$, where we have assumed without lost of generality that $G$ is simply-connected, so $\mathcal{O}_{\rho}$ is $G$ equivariant, cf. footnote 6 . Then we have $i_{\alpha}^{!}(\mathcal{V})[1] \cong i_{\alpha}^{*}(\mathcal{V}(-\alpha))=i_{\alpha}^{*}\left(\mathcal{V}_{\lambda-\rho}(-\alpha+2 \rho)\right)$, where we have dropped the $\mathbb{G}_{m^{-}}$ equivariance to unburden the notation. The vector bundle $i_{\alpha}^{*}\left(\mathcal{V}_{\lambda-\rho}(-\alpha+2 \rho)\right)$ is trivial on the fibers of $\tilde{\pi}_{\alpha}$, since $\langle\check{\alpha},-\alpha+2 \rho\rangle=0$. Thus we have a distinguished triangle

$$
F_{\alpha}(\mathcal{V}) \rightarrow \mathcal{V} \rightarrow \mathcal{V}(-\alpha, 2)
$$

On the other hand, the above filtration on $\mathcal{V}_{\mu}$ yields a filtration on $\mathcal{V}$ and on $\mathcal{V}(-\alpha, 2)$. In both cases all but one terms in this filtration lie in $D_{\operatorname{conv}^{0}(\lambda)}^{G \times \mathbb{G}_{m}}(\tilde{\mathcal{N}})$. The only term which does not is $\mathcal{O}_{\lambda, 0}$ for $\mathcal{V}$, and $\mathcal{O}_{s_{\alpha}(\lambda), 2}$ for $\mathcal{V}(-\alpha, 2)$. Using the induction assumption we get the first isomorphism for $\lambda \in W_{f}(\mu)$.

The second isomorphism is proved similarly, using the observation that for $\mathcal{V}=$ $\mathcal{V}_{\lambda-\rho}(\rho-\alpha)$ and $\mathcal{W}=i_{\alpha}^{*}(\mathcal{V})(-\rho)$ we have $\mathcal{W} \leftrightharpoons \pi_{\alpha}^{!} \tilde{\pi}_{\alpha *} \mathcal{W}$.

Proof of Proposition \%. We prove the statements about $\nabla^{\lambda}$, the proof of the "graded version" is similar. Set $\left(\nabla^{\prime}\right)^{\lambda}=\Pi_{\lambda}^{l}\left(\mathcal{O}_{\lambda}\right)$.

We first check that if $s_{\alpha}(\lambda) \prec \lambda$, then we have canonical distinguished triangles

$$
\begin{aligned}
& F_{\alpha}\left(\left(\nabla^{\prime}\right)^{\lambda}\right) \stackrel{\operatorname{can}_{\alpha}}{\longrightarrow}\left(\nabla^{\prime}\right)^{\lambda} \rightarrow\left(\nabla^{\prime}\right)^{s_{\alpha}(\lambda)},
\end{aligned}
$$

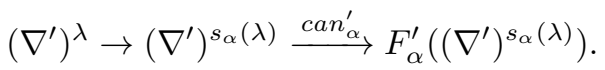


This follows from Lemmas 6, 7: Lemma 7(b) together with Lemma 6 show that $\Pi_{\lambda}^{l}$ commutes with $F_{\alpha}, F_{\alpha}^{\prime}$, then the distinguished triangles follow from Lemma $7(\mathrm{a})$.

It remains to show that $\left(\nabla^{\prime}\right)^{\lambda} \cong \nabla^{\lambda}$.

It suffices to check that

$$
\begin{gathered}
\left(\nabla^{\prime}\right)^{\lambda} \cong \mathcal{O}_{\lambda} \quad \bmod D_{<_{\text {compl }} \lambda}^{G}, \\
\operatorname{Hom}^{\bullet}\left(\left(\nabla^{\prime}\right)^{\lambda},\left(\nabla^{\prime}\right)^{\mu}\right)=0 \quad \text { if } \quad \lambda<_{\text {compl }} \mu,
\end{gathered}
$$

where $D_{<_{\text {compl } l} \lambda}^{G}=D_{\left\{\mu \mid \mu<_{\text {compl } l} \lambda\right\}}^{G}$.

Here the first isomorphism is clear from the definition of $\left(\nabla^{\prime}\right)^{\lambda}$ and the requirement (12) on the order $\leq_{c o m p l}$. The second isomorphism follows from the following stronger statement:

$$
\operatorname{Hom}\left(\left(\nabla^{\prime}\right)^{\lambda},\left(\nabla^{\prime}\right)^{\mu}\right) \neq 0 \Rightarrow \mu \in \operatorname{conv}^{0}(\lambda) \bigvee\left(\mu \in W_{f}(\lambda) \& \mu \preceq \lambda\right)
$$

We now prove (15). Assume first $\lambda \in W_{f}(\mu)$ and $\mu \npreceq \lambda$; we show that in this case

$$
\operatorname{Hom}\left(\left(\nabla^{\prime}\right)^{\lambda},\left(\nabla^{\prime}\right)^{\mu}\right)=0 .
$$

We have $\operatorname{Hom}\left(\left(\nabla^{\prime}\right)^{\lambda},\left(\nabla^{\prime}\right)^{\mu}\right)=\operatorname{Hom}\left(\mathcal{O}_{\lambda},\left(\nabla^{\prime}\right)^{\mu}\right)$, because $\left(\nabla^{\prime}\right)^{\lambda} \cong \mathcal{O}_{\lambda}$ $\bmod D_{c o n v^{0}(\lambda)}^{G}$ and $\left(\nabla^{\prime}\right)^{\mu} \in D_{c o n v^{0}(\lambda)}^{G}(\tilde{\mathcal{N}})^{\perp}$. On the other hand, Lemma 5 implies that $\left(\nabla^{\prime}\right)^{\mu} \cong \mathcal{O}^{\mu} \bmod D_{\succ \mu}^{G}$, where $D_{\succ \mu}^{G}(\tilde{\mathcal{N}})=D_{\{\nu \mid \nu \succ \mu\}}^{G}(\tilde{\mathcal{N}})$. Lemma 5 shows also that $\mathcal{O}_{\lambda} \in \in^{\perp} D_{\succeq \mu}^{G}(\tilde{\mathcal{N}})$, which yields $(16)$.

It remains to show (16) assuming that $\lambda \notin \operatorname{conv}(\mu)$. It is enough to check that $\left(\nabla^{\prime}\right)^{\mu} \in\left(D_{\operatorname{conv}(\lambda)}^{G}\right)^{\perp}$. If $\mu \in \Lambda^{+}$, then $\left(\nabla^{\prime}\right)^{\lambda}=\mathcal{O}_{\lambda}$, so $\left(\nabla^{\prime}\right)^{\mu} \in\left(D_{\operatorname{conv}(\lambda)}^{G}\right)^{\perp}$ by Lemma 5. Comparing (14) with Lemma 7(b) and Lemma 6(b) we see that if $\left(\nabla^{\prime}\right)^{\mu} \in\left(D_{\operatorname{conv}(\lambda)}^{G}\right)^{\perp}$ and $s_{\alpha}(\mu) \prec \mu$, then also $\left(\nabla^{\prime}\right)^{s_{\alpha}(\mu)} \in\left(D_{\operatorname{conv}(\lambda)}^{G}\right)^{\perp}$. Thus $\left(\nabla^{\prime}\right)^{\mu} \in\left(D_{\operatorname{conv}(\lambda)}^{G}\right)^{\perp}$ for all $\mu$ such that $\lambda \notin \operatorname{conv}(\mu)$.

Definition 1. The exotic $t$-structure on $D^{G}(\tilde{\mathcal{N}})$ (respectively $\left.D^{G \times \mathbb{G}_{m}}(\tilde{\mathcal{N}})\right)$ is the $t$-structure of the (graded) exceptional set $\nabla^{\lambda}\left(\right.$ resp. $\left.\nabla^{\lambda, 0}\right)$.

We let $\mathcal{E}^{G}(\tilde{\mathcal{N}}), \mathcal{E}^{G \times \mathbb{G}_{m}}(\tilde{\mathcal{N}})$ denote the hearts of the exotic $t$-structures on $D^{G}(\tilde{\mathcal{N}})$, $D^{G \times \mathbb{G}_{m}}(\tilde{\mathcal{N}})$ respectively. In view of Propositions 2, 4 (see section 2.1.3), isomorphism classes of irreducible objects in $\mathcal{E}^{G}(\tilde{\mathcal{N}})$ are in bijection with $\Lambda$, while irreducible objects in $\mathcal{E}^{G \times \mathbb{G}_{m}}(\tilde{\mathcal{N}})$ are in bijection with $\Lambda \times \mathbb{Z}$. For $\lambda \in \Lambda, n \in \mathbb{Z}$ we let $E_{\lambda} \in \mathcal{E}^{G}(\tilde{\mathcal{N}}), E_{\lambda, n} \in \mathcal{E}^{G \times \mathbb{G}_{m}}(\tilde{\mathcal{N}})$ be the corresponding irreducible objects. It is clear from the construction that the forgetful functor $D^{G \times \mathbb{G}_{m}}(\tilde{\mathcal{N}}) \rightarrow D^{G}(\tilde{\mathcal{N}})$ sends $E_{\lambda, n}$ to $E_{\lambda}$.

2.4. Exotic and perverse. In this subsection we restrict attention to $D^{G}(\tilde{\mathcal{N}})$ to simplify notations; we leave it as an exercise for the reader to provide the "graded" analogue.

Lemma 8. For $\lambda \in \Lambda^{+}$and any $w \in W_{f}$ we have $\pi_{*}\left(\nabla^{w(\lambda)}\right)=A_{\lambda}, \pi_{*}\left(\Delta_{w(\lambda)}\right)=$ $A_{w_{o}(\lambda)}$. 
Proof. We prove the first isomorphism, the proof of the second one is similar.

If $w=1$, then the isomorphism is just the definition of $A_{\lambda}$. Thus we will be done if we show that $\pi_{*}\left(\nabla^{s_{\alpha}(\lambda)}\right) \cong \pi_{*}\left(\nabla^{\lambda}\right)$ for any simple reflection $s_{\alpha}$ and $\lambda \in \Lambda$. This follows from Proposition 7 (b) in view of $\pi_{*} \circ F_{\alpha}=0$. The latter equality is a consequence of the fact that for any $\mathcal{F}$ the complex of sheaves $F_{\alpha}(\mathcal{F})$ is concentrated on $\tilde{\mathcal{N}}_{\alpha}$ and its restriction to any fiber of $\widetilde{\pi}_{\alpha}$ is isomorphic to a sum of several copies of $\mathcal{O}_{\mathbb{P}^{1}}(-1)[d]$ for some $d$; thus its derived global sections vanish.

Corollary 1. The functor $\pi_{*}: D^{G}(\tilde{\mathcal{N}}) \rightarrow D^{G}(\mathcal{N})$ is t-exact, where $D^{G}(\tilde{\mathcal{N}})$ is equipped with the exotic, and $D^{G}(\mathcal{N})$ with the perverse coherent $t$-structure of the middle perversity (see section 2.2).

Proof. Compare the definition of the exotic t-structure with (6) and Lemma 8. $\square$

Proposition 8. For $\lambda \in-\Lambda^{+}$we have $\pi_{*}\left(E_{\lambda}\right)=I C_{O_{w_{o}(\lambda)}, \mathcal{L}_{w_{o}(\lambda)}}$.

For $\lambda \notin-\Lambda^{+}$we have $\pi_{*}\left(E_{\lambda}\right)=0$.

Proof. Recall that for $\lambda \in \Lambda$ the object $E_{\lambda}$ is the image of the unique (up to a constant) non-zero morphism $C_{\lambda}: \Delta_{\lambda} \rightarrow \nabla^{\lambda}$, while for $\lambda \in \Lambda^{+}$the object $I C_{O_{\lambda}, \mathcal{L}_{\lambda}}$ is the image of a unique up to a constant non-zero morphism $c_{\lambda}: A_{w_{o}(\lambda)} \rightarrow A_{\lambda}$. By Lemma 8 and Corollary $1, \pi_{*}\left(E_{\lambda}\right)$ is the image of the morphism $\pi_{*}\left(C_{\lambda}\right): A_{\lambda} \rightarrow$ $A_{w_{o}(\lambda)}$ if $\lambda \in-\Lambda^{+}$. Thus it suffices to show that $\pi_{*}\left(C_{\lambda}\right) \neq 0$ iff $\lambda \in-\Lambda^{+}$.

Assume first that $\lambda \in-\Lambda^{+}$. We have $E_{\lambda} \cong \nabla_{\lambda} \bmod D_{<_{\text {compl }} \lambda}^{G}(\tilde{\mathcal{N}})$. Since $\lambda \in-\Lambda^{+}$, we have $\nu<\lambda \Rightarrow \nu \in \operatorname{conv}_{0}(\lambda)$; we can also assume that the complete order $\leq_{\text {compl }}$ is chosen so that $\nu<_{\text {compl }} \lambda \Rightarrow \nu \in \operatorname{conv}_{0}(\lambda)$. Thus if $\pi_{*}\left(E_{\lambda}\right)=$ 0 , then $\pi_{*}\left(\nabla^{\lambda}\right)=A_{w_{o}(\lambda)}$ lies in the full triangulated subcategory generated by $\pi_{*}\left(\nabla^{\nu}\right), \nu \in \operatorname{conv}^{0}(\lambda)$. In view of Lemma 8 , the latter category is generated by $A_{\nu}$, $\Lambda^{+} \ni \nu \prec w_{o}(\lambda)$. This category does not, in fact, contain $A_{w_{o}(\lambda)}$ by, e.g., (8).

Assume now that $\lambda \notin-\Lambda^{+}$, thus there exists a simple reflection $s_{\alpha}$, such that $s_{\alpha}(\lambda) \prec \lambda$. Consider (the non-graded version of) the first distinguished triangle of Proposition $7(\mathrm{~b})$. We have $\operatorname{Hom}^{\bullet}\left(\Delta_{\lambda}, \nabla^{s_{\alpha}(\lambda)}\right)=0$, thus the morphism $C_{\lambda}$ factors through a morphism $\Delta_{\lambda} \rightarrow F_{\alpha}\left(\nabla^{\lambda}\right)$. It was explained in the proof of Lemma 8 that $\pi_{*} \circ F_{\alpha}=0$. Hence in this case we have $\pi_{*}\left(C_{\lambda}\right)=0$.

2.5. Positivity Lemma. We state the key result, which formally implies our main Theorem 3 ; the proof appears in section 4 .

Lemma 9. $\operatorname{Hom}^{i}\left(\Delta_{\lambda, n+\delta_{\lambda}}, E_{\mu, \delta_{\mu}}\right)=0$ if $i>n$;

$\operatorname{Hom}^{i}\left(E_{\mu, \delta_{\mu}}, \nabla^{\lambda, n+\delta_{\lambda}}\right)=0$ if $i>-n$ (see 1.4 for notations).

Remark 5. The Lemma is equivalent to the statement that some $\mathbb{Z}$-graded vector spaces have trivial components of negative degree, hence the name.

Remark 6. There are several results in representation theory, the Kazhdan-Lusztig conjecture being the first and the most famous one, which are proved by identifying an algebraically defined vector space with a (co)stalk of an irreducible perverse sheaf, and then using the deep information about the action of Frobenius on this (co)stalk provided by Purity Theorem from [BBD]. Our strategy for proving the key Lemma 9 also follows this pattern. More precisely, the proof of Lemma 9 relies on purity of perverse sheaves on the affine flag variety; see also Remark 4 in section 2.1.6 above. 


\section{Quantum group modules: Proof modulo the Positivity Lemma.}

In this section the semi-simple group $G$ is assumed to be of adjoint type.

We start by fixing notations. Recall that irreducible objects of $\mathrm{U}_{q}$-mod are parameterized by their highest weight $\lambda \in \Lambda^{+}$. For $\lambda \in \Lambda^{+}$let $V(\lambda)$ be the corresponding irreducible representation. We have the dot action of the affine Weyl group $W$ on $\Lambda, w: \mu \mapsto w \cdot \mu=w(\mu+\rho)-\rho$ (in particular, the subgroup $\Lambda \subset W$ acts by $\lambda: \mu \mapsto \mu+l \lambda)$. By the linkage principle [APW] the irreducible object $V(\lambda)$ lies in the block $\mathrm{U}_{q}-\bmod ^{0}$ iff $\lambda$ lies in the $W$ orbit of 0 . For $w \in W$ we have $w \cdot 0 \in \Lambda^{+}$iff $\ell(w) \leq \ell\left(w_{f} w\right)$ for all $w_{f} \in W_{f}$. There is exactly one such element in each left coset of $W_{f}$ in $W$. On the other hand, $\Lambda \rightleftharpoons W_{f} \backslash W$. Recall that $w_{\lambda}$ is the minimal length representative of the coset $W_{f} \lambda \subset W$, and set $L_{\lambda}=V\left(w_{\lambda} \cdot 0\right)$. For example, if $\lambda \in \Lambda^{+}$we have $L_{\lambda}=V(l \lambda)$, while for a strictly anti-dominant $\lambda$ we have $L_{\lambda}=V\left(w_{o}(\lambda)-2 \rho\right)$, where $w_{o} \in W_{f}$ is the longest element.

Similarly, we let $\operatorname{Weyl}_{\lambda}=\operatorname{Weyl}\left(w_{\lambda} \cdot 0\right), \operatorname{coWeyl}^{\lambda}=\operatorname{coWeyl}\left(w_{\lambda} \cdot 0\right)$ be the Weyl module and dual Weyl module with highest weight $w_{\lambda} \cdot 0$, and $T_{\lambda}=T\left(w_{\lambda} \cdot 0\right)$ be the corresponding tilting module.

3.1. Some results of $[\mathrm{ABG}]$. The result of $[\mathrm{ABG}]$ yields a functor $\Psi: D^{G \times \mathbb{G}_{m}}(\tilde{\mathcal{N}}) \rightarrow D^{b}\left(\mathrm{U}_{q}-\bmod ^{0}\right)$.

It satisfies the following properties

$$
\begin{gathered}
\Psi(\mathcal{F}(1)) \cong \Psi(\mathcal{F})[1] \\
\Psi: \bigoplus_{n \in \mathbb{Z}} \operatorname{Hom}(\mathcal{F}, \mathcal{G}[n](-n)) \stackrel{\sim}{\longrightarrow} \operatorname{Hom}(\Psi(\mathcal{F}), \Psi(\mathcal{G})) ; \\
\Psi: \mathcal{O}_{\lambda, 0} \mapsto \operatorname{RInd}_{\mathrm{B}_{q}}^{\mathrm{U}_{q}}(l \lambda) ; \\
H\left(b_{q}, \Psi(\mathcal{F})\right) \cong \Gamma\left(\left.\mathcal{F}\right|_{\mathfrak{n}}\right),
\end{gathered}
$$

the latter isomorphism is $B$-equivariant (here we use notation $\Gamma$ for the functor of global sections on $D^{B \times \mathbb{G}_{m}}(\mathfrak{n})$ and we write " $\Gamma$ " rather than " $R \Gamma$ " since $\mathfrak{n}$ is affine, thus the functor of global sections is exact). Also, for $V \in \operatorname{Rep}(G)$ we have

$$
\Psi(\mathcal{F} \otimes V) \cong \Psi(\mathcal{F}) \otimes F r^{*}(V),
$$

where $\operatorname{Fr}: \mathrm{U}_{q} \rightarrow U(\mathfrak{g})$ is Lusztig's quantum Frobenius morphism.

Recall the functors $H, \widetilde{H}$ from the Introduction.

Proposition 9. $H\left(u_{q}, \Psi(\mathcal{F})\right) \cong R \Gamma(\mathcal{F})=\Gamma\left(\pi_{*}(\mathcal{F})\right)$.

Proof. The left hand side can be rewritten as $\bigoplus_{\lambda \in \Lambda^{+}} V_{\lambda} \otimes \operatorname{Hom}_{\mathbf{U}_{q}}(\mathbf{k}, \Psi(\mathcal{F}) \otimes$ $F r^{*}\left(V_{\lambda}^{*}\right)$ ) where $V_{\lambda}$ is an irreducible $U(\mathfrak{g})$ module with highest weight $\lambda$ (see $[\mathrm{AG}]$ ). Using (19) for $\lambda=0$, and (21) we can rewrite it as $\bigoplus_{\lambda} V_{\lambda} \otimes \operatorname{Hom}_{D^{G}(\tilde{\mathcal{N}})}^{\bullet}\left(\mathcal{O}, \mathcal{F} \otimes V_{\lambda}^{*}\right)=$ $\operatorname{Hom}_{D(\tilde{\mathcal{N}})}^{\bullet}(\mathcal{O}, \mathcal{F})=R \Gamma(\mathcal{F})$.

We are ready to give the precise statement of the main result.

Theorem 3. $\Psi\left(E_{\lambda, \delta_{\lambda}}\right) \cong T_{\lambda}$. 
3.2. Consequences of Theorem 3. Recall from section 2.2 perverse IC sheaves $I C_{O, \mathcal{L}} \in D^{G}(\mathcal{N})$ (introduced in [B2]) and the bijection $\lambda \leftrightarrow\left(O_{\lambda}, \mathcal{L}_{\lambda}\right)$ between $\Lambda^{+}$ and pairs $(O, \mathcal{L})$ where $O \subset \mathcal{N}$ is a $G$ orbit and $\mathcal{L}$ is an irreducible $G$-equivariant vector bundle (introduced in [Lc], see also [B2], [B]).

The following result contains a more precise version of Theorem 1.

Corollary 2. a) For $\lambda \in \Lambda$ the sheaf $\widetilde{H}\left(T_{\lambda}\right)$ is isomorphic to the direct sum of all cohomology sheaves of the complex $E_{\lambda}$.

b) For $\lambda \in-\Lambda^{+}$we have

$$
H\left(T_{\lambda}\right) \cong R \Gamma\left(E_{\lambda}\right) \cong \Gamma\left(I C_{O_{w_{o}(\lambda)}}, \mathcal{L}_{w_{o}(\lambda)}\right) .
$$

For $\lambda \notin-\Lambda^{+}$we have

$$
H\left(T_{\lambda}\right) \cong R \Gamma\left(E_{\lambda}\right)=0 .
$$

Proof. a) follows directly from Theorem 3 and (20). The isomorphism $H\left(T_{\lambda}\right) \cong$ $R \Gamma\left(E_{\lambda}\right)$ follows from Theorem 3 and Proposition 9. Then (b) follows from Proposition 8.

Remark 7 . The fact that $H\left(T_{\lambda}\right)=0$ for $\lambda \notin-\Lambda^{+}$, which was deduced above from properties of exotic sheaves, is well-known, see, e.g., [O], §3.2.

Comparing this statement with Proposition 2 of [B] we get the following version of a conjecture by J. Humphreys $[\mathrm{H}]$.

Corollary 3. Let $\lambda \in-\Lambda^{+}$be an anti-dominant weight; let $\underline{c}$ be the 2-sided cell in the affine Weyl group $W$ containing $\lambda$ (where we use the standard embedding $\Lambda \subset W)$. The support of $H\left(T_{\lambda}\right)$ as a coherent sheaf on $\mathcal{N}$ is the closure of the nilpotent orbit $O_{\underline{c}} \subset \mathcal{N}$, which corresponds to $\underline{c}$ via the bijection of $[\mathrm{Lc}]$.

Remark 8. Corollaries 2(b) and 3 imply conjectures in $\S 3.2$ of [O]. Indeed, the classes of $I C_{O, \mathcal{L}}$ in the Grothendieck group $K\left(\mathrm{Coh}^{G \times \mathbb{G}_{m}}(\mathcal{N})\right)$ form the canonical basis of the latter group in the sense of [O]; this is proved in [B].

Example 1. (cf. [O], end of §3.2) When the equivariant vector bundle $\mathcal{L}$ is trivial we have $I C_{O, \mathcal{L}}=j_{*}\left(\mathcal{O}_{O}\right)\left[d_{O}-d\right]=N m_{*}\left(\mathcal{O}_{\hat{O}}\right)\left[d_{O}-d\right]$ where $j: O \hookrightarrow \mathcal{N}$ is the embedding, $j_{*}$ stands for the non-derived direct image, and $N m: \hat{O} \rightarrow \bar{O}$ is the normalization morphism for the closure $\bar{O}$ of $O$ (see [B2], Remark 10). Thus for some indecomposable tilting $T_{\lambda}$ we get $H\left(T_{\lambda}\right)=\mathcal{O}_{\hat{O}}$. In fact, [B], Proposition 1 implies that this happens precisely when $\lambda \in-\Lambda^{+}$is such that the minimal length representative of the two-sided coset $W_{f} \lambda W_{f} \subset W$ is a Duflo involution.

3.3. Proof of Theorem 3 modulo the Positivity Lemma. We start with

Lemma 10. $\Psi\left(\nabla_{\lambda, 0}\right) \cong \operatorname{coWeyl}^{\lambda}[-\delta(\lambda)], \Psi\left(\Delta_{\lambda, 0}\right) \cong \operatorname{Weyl}_{\lambda}[-\delta(\lambda)]$.

Proof. We prove the first isomorphism, the second one is similar.

Set $D_{<\lambda}^{b}\left(\mathrm{U}_{q}-\bmod ^{0}\right)=\ll L_{\mu} \mid \mu<\lambda \gg$, and define $D_{<\text {compl } \lambda}^{b}\left(\mathrm{U}_{q}-\bmod ^{0}\right)$ similarly. Recall the Quantum Weak Borel-Weil Theorem of [APW], which implies that

$$
\operatorname{RInd}_{\mathrm{B}_{q}}^{\mathrm{U}_{q}}(l \lambda) \cong L_{\lambda}\left[-\delta_{\lambda}\right] \cong \operatorname{coWeyl}^{\lambda}\left[-\delta_{\lambda}\right] \bmod D_{<\lambda}^{b}\left(\mathrm{U}_{q}-\bmod ^{0}\right) .
$$

Using (19) we see that $\Psi\left(\mathcal{O}_{\lambda, 0}\right) \cong \operatorname{coWeyl}^{\lambda}[-\delta(\lambda)] \bmod D_{<\lambda}^{b}\left(\mathrm{U}_{q}-\bmod ^{0}\right)$. 
It follows by induction in $\lambda$ that $D_{\leq \lambda}^{b}\left(\mathrm{U}_{q}-\bmod ^{0}\right)=\Psi\left(D_{\leq \lambda}^{G \times \mathbb{G}_{m}}(\tilde{\mathcal{N}})\right)$, where $D_{\leq \lambda}^{G \times \mathbb{G}_{m}}(\tilde{\mathcal{N}})=\ll \mathcal{O}_{\mu, n} \mid \mu<\lambda \gg$, and similarly with $\leq$ replaced by $\leq_{\text {compl }}$ or $<_{\text {compl }}$. Recall that by the definition of $\nabla^{\lambda, n}$ we have

$$
\begin{gathered}
\nabla^{\lambda, 0} \in D_{<\text {compl }}^{G \times \mathbb{G}_{m}}(\tilde{\mathcal{N}})^{\perp}, \\
\nabla^{\lambda, 0} \cong \mathcal{O}_{\lambda, 0} \quad \bmod D_{<\text {compl }}^{G \times \mathbb{G}_{m}}(\tilde{\mathcal{N}}) .
\end{gathered}
$$

In view of (18) this implies:

$$
\begin{gathered}
\Psi\left(\nabla^{\lambda, 0}\right) \in D_{<\text {compl }}\left(\mathrm{U}_{q}-\bmod ^{0}\right)^{\perp} \\
\Psi\left(\nabla^{\lambda, 0}\right) \cong L_{\lambda}\left[-\delta_{\lambda}\right] \cong \operatorname{coWeyl}^{\lambda}\left[-\delta_{\lambda}\right] \quad \bmod D_{<_{c o m p l}}^{b}\left(\mathrm{U}_{q}-\bmod ^{0}\right) .
\end{gathered}
$$

It follows that both $\Psi\left(\nabla^{\lambda, 0}\right)\left[\delta_{\lambda}\right]$ and coWeyl ${ }^{\lambda}$ are injective hulls of $L_{\lambda}$ in the Serre subcategory of $\mathrm{U}_{q}$-mod ${ }^{0}$ generated by $L_{\mu}$ with $\mu \leq_{\text {compl }} \lambda$; hence these two objects are isomorphic.

We are now ready to prove Theorem 3 .

Comparing the Positivity Lemma 9 with (17) and (18) we see that

$$
\operatorname{Hom}^{k}\left(\operatorname{Weyl}_{\mu}, \Psi\left(E_{\lambda, \delta_{\lambda}}\right)\right)=\bigoplus_{i+n=-k} \operatorname{Hom}^{i}\left(\Delta_{\mu, n+\delta_{\mu}}, E_{\lambda, \delta_{\lambda}}\right)=0
$$

for $k>0$, and similarly $\operatorname{Hom}^{k}\left(\Psi\left(E_{\lambda, \delta_{\lambda}}\right)\right.$, coWeyl $\left.{ }^{\mu}\right)=0$ for $k>0$. By Lemma 4 this implies that $\Psi\left(E_{\lambda, \delta_{\lambda}}\right)$ is a tilting object of $\mathrm{U}_{q}-\bmod ^{0}$.

The endomorphism algebra $\operatorname{End}\left(\Psi\left(E_{\lambda, \delta_{\lambda}}\right)\right) \cong \operatorname{Hom}^{\bullet}\left(E_{\lambda}, E_{\lambda}\right)$ carries a grading by non-negative integers, with a one-dimensional component of degree zero (namely, the homological grading). Hence it has no nontrivial idempotents, thus $\Psi\left(E_{\lambda, \delta_{\lambda}}\right)$ is indecomposable. Finally, (the proof of) Lemma 10 implies that $\Psi\left(E_{\lambda, \delta_{\lambda}}\right) \in$ $D_{\leq_{\text {compl }} \lambda}^{b}\left(\mathrm{U}_{q}-\bmod ^{0}\right)$, but $\Psi\left(E_{\lambda, \delta_{\lambda}}\right) \notin D_{<_{\text {compl }} \lambda}^{b}\left(\mathrm{U}_{q}-\bmod ^{0}\right)$, hence $\Psi\left(E_{\lambda, \delta_{\lambda}}\right) \cong T_{\lambda}$.

\section{Constructible sheaves on affine flags: proof of Positivity Lemma.}

In this section $G$ is an arbitrary semi-simple group.

The objective of this section is to express the Hom spaces in Lemma 9 as eigenspaces of Frobenius acting on a (co)stalk of an IC sheaf; this is achieved in Corollary 6.

4.1. Perverse sheaves on affine flags for $G^{\sim}$. Recall some results of $[\mathrm{AB}] .{ }^{7}$ Fix $q=p^{a}$ where $p$ is a prime number. Consider the Langlands dual group $G^{\curvearrowleft}$ over the base field $\overline{\mathbb{F}}_{q}$; the "loop" group ind-scheme $\mathbf{G}_{\overline{\mathbb{F}}_{\mathbf{q}}((\mathbf{t}))}$ and its group subschemes $\mathbf{G}^{\circ} \mathbf{O} \supset \mathbf{I}, \mathbf{I}^{-}$; thus we have $\mathbf{G}_{\overline{\mathbb{F}}_{\mathbf{q}}((\mathbf{t}))}\left(\overline{\mathbb{F}}_{q}\right)=G^{\sim}\left(\overline{\mathbb{F}}_{q}((t))\right), \mathbf{G}^{\sim} \mathbf{O}\left(\overline{\mathbb{F}}_{q}\right)=G^{\sim}(O)$, $\mathbf{I}\left(\overline{\mathbb{F}}_{q}\right)=I, \mathbf{I}^{-}\left(\overline{\mathbb{F}}_{q}\right)=I^{-}$where $O=\overline{\mathbb{F}}_{q}[[t]]$, and $I, I^{-}$are opposite Iwahori subgroups in $G^{\smile}\left(\overline{\mathbb{F}}_{q}((t))\right)$. The affine flag variety (ind-scheme) $\mathcal{F} \ell$ is the homogeneous space $\mathbf{G}_{\overline{\mathbb{F}}_{\mathbf{q}}((\mathbf{t}))} / \mathbf{I}$.

\footnotetext{
${ }^{7}$ Notice a discrepancy in notations between $[\mathrm{AB}]$ and the present paper: the group denoted by $G$ here corresponds to $G^{\sim}$ of $[\mathrm{AB}]$, and vice versa.
} 
4.1.1. Iwahori-Whittaker sheaves and equivalence $\Phi$. Let $\mathcal{P}_{\text {asph }}$ denote the category of Iwahori-Whittaker perverse sheaves; this is a subcategory in the category of perverse $l$-adic sheaves on $\mathcal{F} \ell$ defined in [AB], $\S 2.5$ (it is denoted $\mathcal{P}_{\mathcal{I} \mathcal{W}}$ in loc. cit.); it consists of perverse sheaves, which "transform as a non-degenerate character" under the action of the pro-unipotent radical of the Iwahori group $\mathbf{I}^{-}$. The group scheme $\mathbf{G}^{-} \mathbf{O}$ acts on $\mathcal{F} \ell$, and the orbits of this action are indexed by the set $W_{f} \backslash W \leftleftarrows \Lambda$. For $\lambda \in \Lambda$ let $\mathcal{F} \ell^{\lambda}$ be the corresponding $\mathbf{G}^{\circ}$ o orbit, and $i_{\lambda}: \mathcal{F} \ell^{\lambda} \hookrightarrow \mathcal{F} \ell$ be the embedding.

For $\lambda \in \Lambda$ there is a unique (up to an isomorphism) irreducible object $I C_{\lambda}^{e t} \in$ $\mathcal{P}_{\text {asph }}$ whose support is the closure of $\mathcal{F} \ell^{\lambda}$. Also there is a unique standard object $\Delta_{\lambda}^{e t}$ (denoted by $\Delta_{w_{\lambda}}$ in $[\mathrm{AB}]$ ) characterized by $i_{\mu}^{*}\left(\Delta_{\lambda}^{e t}\right)=0$ for $\mu \neq \lambda$, $i_{\lambda}^{*}\left(\Delta_{\lambda}^{e t}\right) \cong i_{\lambda}^{*}\left(I C_{\lambda}^{e t}\right)$ and a unique costandard object $\nabla_{e t}^{\lambda}$ (denoted by $\nabla_{w_{\lambda}}$ in loc. cit.) characterized by $i_{\mu}^{!}\left(\nabla_{e t}^{\lambda}\right)=0$ for $\mu \neq \lambda$, $i_{\lambda}^{*}\left(\nabla_{e t}^{\lambda}\right) \cong i_{\lambda}^{*}\left(I C_{\lambda}^{e t}\right)$. (The sub or superscript "et" (for etale) is used to remind that we are in a context different from that of section 2). For $\mathcal{F} \in D^{b}\left(\mathcal{P}_{\text {asph }}\right)$, and a point $x_{\lambda} \in \mathcal{F} \ell^{\lambda}$, which lies in the open orbit of an opposite Iwahori subgroup $\mathbf{I}^{-}$we have

$$
\begin{aligned}
& \operatorname{Hom}^{\bullet}\left(\Delta_{\lambda}^{e t}, \mathcal{F}\right)=\iota_{\lambda}^{!}(\mathcal{F})\left[d_{\lambda}\right] \\
& \operatorname{Hom}^{\bullet}\left(\mathcal{F}, \nabla_{\lambda}^{e t}\right)=\left(\iota_{\lambda}^{*}(\mathcal{F})\left[-d_{\lambda}\right]\right)^{*},
\end{aligned}
$$

where $\iota_{\lambda}: x_{\lambda} \hookrightarrow \mathcal{F} \ell$ is the embedding, and $d_{\lambda}=\operatorname{dim} \mathcal{F} \ell^{\lambda}$.

The objects $\Delta_{\lambda}^{e t}, \nabla_{e t}^{\lambda}$ can be described as extension by zero (respectively, direct image) of an Artin-Schreier sheaf on $\mathcal{F} \ell^{\lambda}$; for future reference we spell this out for $\lambda=0$. Let $B^{\sim}, B^{\sim}$ - be the images under the projection $\mathbf{G}^{\sim} \mathbf{O} \rightarrow G^{\sim}$ of the Iwahori subgroups I, I- respectively; let $N^{\sim} \subset B_{-}^{\sim}$ be the unipotent radical. Let $j: N^{\sim} \hookrightarrow G^{\sim} / B^{\sim}$ be given by $n \mapsto n B^{\sim}$; thus $j$ is an open embedding. Let $\psi: N^{\sim} \rightarrow \mathbb{A}^{1}$ be a non-degenerate character. Define the Whittaker sheaf on $G^{\triangleleft} / B^{\curvearrowright}$ by

$$
W h=j_{*} \psi^{*}(A S)\left[\operatorname{dim} G^{\sim} / B^{`}\right] \cong j_{!} \psi^{*}(A S)\left[\operatorname{dim} G^{\sim} / B^{`}\right],
$$

where $A S$ denotes the Artin-Schreier sheaf. Then $\Delta_{0}^{e t} \cong \nabla_{e t}^{0}$ is the direct image of $W h$ under the closed embedding $i_{0}: \mathcal{F} \ell^{0}=G^{\sim} / B^{\sim} \hookrightarrow \mathcal{F} \ell$.

One of the main results of $[\mathrm{AB}]$ is an equivalence of triangulated categories

$$
\Phi: D^{G}(\tilde{\mathcal{N}}) \cong D^{b}\left(\mathcal{P}_{\text {asph }}\right) .
$$

4.1.2. I-equivariant sheaves. Let $D_{\mathbf{I}}(\mathcal{F} \ell)$ be the I-equivariant derived category of $l$-adic sheaves on $\mathcal{F} \ell$, and $\mathcal{P}_{\mathbf{I}} \subset D_{\mathbf{I}}(\mathcal{F} \ell)$ be the subcategory of perverse sheaves. Convolution of equivariant complexes provides $D_{\mathbf{I}}(\mathcal{F} \ell)$ with a structure of monoidal category, and it provides $D^{b}\left(\mathcal{P}_{\text {asph }}\right)$ with a structure of a (right) module category; ${ }^{8}$ we denote convolution of complexes $\mathcal{F} \in D^{b}\left(\mathcal{P}_{\text {asph }}\right), \mathcal{G} \in D_{\mathbf{I}}(\mathcal{F} \ell)$ by $\mathcal{F} * \mathcal{G}$.

The equivalence $\Phi$ is obtained as a composition $\Phi=A v_{\mathbf{I}^{-}, \psi} \circ F$. Here $F$ is a certain (monoidal) functor $D^{G}(\tilde{\mathcal{N}}) \rightarrow D_{\mathbf{I}}(\mathcal{F} \ell)$ and $A v_{\mathbf{I}^{-}, \psi}$ is the functor of "averaging against a character" $\psi$, i.e., $A v_{\mathbf{I}^{-}, \psi}: \mathcal{F} \mapsto \Delta_{0}^{e t} * \mathcal{F}$.

We have $F\left(\mathcal{O}_{\lambda}\right)=J_{\lambda}$ where $J_{\lambda}$ is the Wakimoto sheaf, see [AB], [ABG], [GH] and $F\left(V_{\lambda} \otimes \mathcal{O}_{\tilde{\mathcal{N}}}\right)=Z_{\lambda}$ is the central sheaf constructed in [G].

Also, it is proved in $[\mathrm{AB}]$ that $A v_{\mathbf{I}^{-}, \psi}: \mathcal{P}_{I} \rightarrow \mathcal{P}_{a s p h}$, i.e. $A v_{\mathbf{I}^{-}, \psi}$ is $t$-exact.

\footnotetext{
${ }^{8}$ Here we use that $D^{b}\left(\mathcal{P}_{a s p h}\right)$ is identified with a full subcategory in the derived category of constructible sheaves on $\mathcal{F} \ell$, cf. [AB], Lemma 1 .
} 
The $\mathbf{I}$ orbits in $\mathcal{F} \ell$, also called Schubert cells, are indexed by $W$; for $w \in W$ let $\mathcal{S}_{w}$ denote the corresponding Schubert cell, and $j_{w}: \mathcal{S}_{w} \hookrightarrow \mathcal{F} \ell$ be the embedding. We have $J_{\lambda} \in \mathcal{P}_{I}$, the support of $J_{\lambda}$ is the closure of $\mathcal{S}_{\lambda}$, and we have

$$
\begin{aligned}
& \operatorname{supp}\left(A v_{I^{-}, \psi}\left(J_{\lambda}\right)\right)=\overline{\mathcal{F} \ell^{\lambda}} \\
& \left.\left.A v_{I^{-}, \psi}\left(J_{\lambda}\right)\right|_{\mathcal{F} \ell^{\lambda}} \cong \Delta_{\lambda}^{e t}\right|_{\mathcal{F} \ell^{\lambda}},
\end{aligned}
$$

where supp stands for "support", and $\overline{\mathcal{F} \ell^{\lambda}}$ is the closure of $\mathcal{F} \ell^{\lambda}$. For $w \in W$ set $j_{w !}=j_{w !}\left(\overline{\mathbb{Q}_{l}}[\ell(w)]\right), j_{w *}=j_{w *}\left(\overline{\mathbb{Q}_{l}}[\ell(w)]\right)$.

We will need another simple geometric property of the Wakimoto sheaves.

Lemma 11. We have $i_{\lambda}^{*}\left(J_{\lambda}\right) \cong i_{\lambda}^{*}\left(j_{\lambda !}\right)$.

Proof. It suffices to show that $\operatorname{Hom}^{\bullet}\left(J_{\lambda}, j_{w *}\right)=0$ for $w \in W_{f} \lambda, w \neq \lambda$. Let $w_{\max } \in W$ be the maximal length element of the coset $W_{f} \lambda$, and set $w_{1}=$ $w_{\text {max }} w^{-1}$. We have $w \in W_{f}$ and $\ell\left(w_{1} w\right)=\ell\left(w_{1}\right)+\ell(w)$. It follows that $j_{w_{1}} * j_{w *}=$ $j_{w_{\text {max }} *}$. Since the functor $\mathcal{F} \mapsto j_{w_{1} *} * \mathcal{F}$ is an equivalence (with inverse equivalence given by $\mathcal{F} \mapsto j_{w_{1}}$ ! $\left.* \mathcal{F}\right)$, it is enough to check that $\operatorname{Hom}^{\bullet}\left(j_{w_{1} *} * J_{\lambda}, j_{w_{\max } *}\right)=0$. Thus we will be done if we check that the (closure of the) support of $j_{w_{1} *} * J_{\lambda}$ does not contain $\mathcal{S}_{w_{\max }}$. We claim that in fact this support coincides with the closure of $\mathcal{S}_{w_{1} \lambda}$, which clearly does not contain $\mathcal{S}_{w_{\max }}$. To check that $\operatorname{supp}\left(j_{w_{1} *} * J_{\lambda}\right)=\overline{\mathcal{S}_{w_{1} \lambda}}$ we check that $j_{w_{1} *} * J_{\lambda}$ is a perverse sheaf, and that the Euler characteristic of its stalk at a point $x \in \mathcal{F} \ell$ equals 1 if $x \in \mathcal{S}_{w_{1} \lambda}$, and 0 otherwise. The claim about the Euler characteristic is standard. To prove that $j_{w_{1}} * J_{\lambda}$ is perverse we use a trick due to Mirković (cf. [AB], Appendix 6.1). Recall that if $\lambda=\mu_{1}-\mu_{2}$, where $\mu_{1}, \mu_{2} \in \Lambda^{+}$, then $J_{\lambda}=j_{\mu_{1} *} * j_{-\mu_{2} !}$. Since $w_{1} \in W_{f}, \mu \in \Lambda^{+}$we have $\ell\left(w_{1} \mu_{1}\right)=\ell\left(w_{1}\right)+\ell\left(\mu_{1}\right)$. Thus we have $j_{w_{1} *} * J_{\lambda}=j_{w_{1} *} * j_{\mu_{1} *} * j_{-\mu_{2}}$ ! $=j_{w_{1} \mu_{1} *} * j_{-\mu_{2}}$ ! . The convolution $j_{u *} * j_{v !}$ is perverse for any $u, v \in W$, see $[\mathrm{AB}]$, Appendix 6.1.

\subsubsection{Exotic coherent and perverse constructible sheaves.}

Lemma 12. $\Phi\left(\nabla^{\lambda}\right) \cong \nabla_{e t}^{\lambda}, \Phi\left(\Delta_{\lambda}\right) \cong \Delta_{\lambda}^{e t}$.

Proof. Let $D_{\preceq \lambda}^{b}\left(\mathcal{P}_{\text {asph }}\right)$ (respectively, $D_{\prec \lambda}^{b}\left(\mathcal{P}_{\text {asph }}\right)$ ) be the subcategory of complexes supported on $\overline{\mathcal{F} \ell^{\lambda}}$ (respectively, on $\overline{\mathcal{F} \ell^{\lambda}} \backslash \mathcal{F} \ell^{\lambda}$ ). In view of (23) we get, by induction in $\lambda$, that

$$
D_{\preceq \lambda}^{b}\left(\mathcal{P}_{a s p h}\right)=\ll A v_{I^{-}, \psi}\left(J_{\lambda}\right) \gg ;
$$

since $A v_{I^{-}, \psi}\left(J_{\lambda}\right)=\Phi\left(\mathcal{O}_{\lambda}\right)$, we have $D_{\preceq \lambda}^{b}\left(\mathcal{P}_{a s p h}\right)=\Phi\left(D_{\preceq \lambda}^{G}(\tilde{\mathcal{N}})\right)$; and similarly with $\preceq$ replaced by $\prec$. Thus $\Phi\left(\nabla_{\lambda}\right) \in D_{\prec \lambda}^{b}\left(\mathcal{P}_{\text {asph }}\right)^{\perp} \cap D_{\preceq \lambda}^{b}\left(\mathcal{P}_{\text {asph }}\right)$, which means that $\Phi\left(\nabla^{\lambda}\right)$ is supported on $\overline{\mathcal{F} \ell^{\lambda}}$ and its "shriek" restriction to the boundary of $\mathcal{F} \ell^{\lambda}$ vanishes. Also we have

$$
\Phi\left(\nabla_{\lambda}\right) \cong \Phi\left(\mathcal{O}_{\lambda}\right) \quad \bmod D_{\prec \lambda}^{b}\left(\mathcal{P}_{a s p h}\right)
$$

which means that $\left.\left.\Phi\left(\nabla^{\lambda}\right)\right|_{\mathcal{F} \ell^{\lambda}} \cong A v_{I^{-}, \psi}\left(J_{\lambda}\right)\right|_{\mathcal{F} \ell^{\lambda}}$. Thus we are done by $(23)$.

Corollary 4. $\Phi$ sends the exotic t-structure to the tautological one and $\Phi\left(E_{\lambda}\right) \cong$ $I C_{\lambda}^{e t}$.

Proof. Compare the definition of the exotic $t$-structure with Remark 2. Also observe that $E_{\lambda}$ is the image of the unique (up to a constant) non-zero morphism $\Delta_{\lambda} \rightarrow \nabla^{\lambda}$, while $I C_{\lambda}^{e t}$ is the image of the unique morphism $\Delta_{\lambda}^{e t} \rightarrow \nabla_{e t}^{\lambda}$. 
Corollary 5. For $\mathcal{F} \in D^{G}(\tilde{\mathcal{N}})$ we have: $\operatorname{Hom}^{\bullet}\left(\Delta_{\lambda}, \mathcal{F}\right) \cong \iota_{\lambda}^{*}(\Phi(\mathcal{F}))$; $\operatorname{Hom}^{\bullet}\left(\mathcal{F}, \nabla^{\lambda}\right)^{*} \cong \iota_{\lambda}^{!}(\Phi(\mathcal{F}))$.

4.2. Frobenius weights. By a Weil complex on a scheme $X$ defined over $\mathbb{F}_{q}$ we will mean an object $\mathcal{F}$ in the "derived category" of $l$-adic sheaves on $X_{\overline{\mathbb{F}}_{q}}$ equipped with an isomorphism $F r^{*}(\mathcal{F}) \cong \mathcal{F}$; a Weil perverse sheaf is a Weil complex, which is also a perverse sheaf. By [BBD], Proposition 5.1.2, the category of Weil perverse sheaves on $X$ contains the category of perverse sheaves on the scheme over $\mathbb{F}_{q}$ as a full subcategory.

Recall the compatibility of $F$ with Frobenius. Let $\mathbf{q}: \tilde{\mathcal{N}} \rightarrow \tilde{\mathcal{N}}$ be the multiplication by $q$ map, $\mathbf{q}:(\mathfrak{b}, x) \mapsto(\mathfrak{b}, q x)$. Then we have (see $[\mathrm{AB}]$, Proposition $1)$ :

$$
F \circ F r^{*} \cong \mathbf{q}^{*} \circ F .
$$

Fix a square root of $q, q^{1 / 2} \in \overline{\mathbb{Q}_{l}}$. Then for $\mathcal{F} \in D^{G \times \mathbb{G}_{m}}(\tilde{\mathcal{N}})$ the $\mathbb{G}_{m}$-equivariant structure induces an isomorphism of objects in $D^{G}(\tilde{\mathcal{N}})$ :

$$
\mathbf{q}^{*}(\mathcal{F}) \cong \mathcal{F}
$$

(recall that the action of $\mathbb{G}_{m}$ on $\tilde{\mathcal{N}}$ is given by $t:(\mathfrak{b}, x) \mapsto\left(\mathfrak{b}, t^{2} x\right)$ ).

By means of (24) we get an isomorphism

$$
F r^{*}(F(\mathcal{F})) \cong F(\mathcal{F}) \text {. }
$$

Thus we get a functor $\tilde{F}$ from $D^{G \times \mathbb{G}_{m}}(\tilde{\mathcal{N}})$ to the category of Weil complexes on $\mathcal{F} \ell$.

Notice that the choice of $q^{1 / 2}$ defines also a square root $\mathcal{F} \mapsto \mathcal{F}\left(\frac{1}{2}\right)$ of the functor of Tate twist on the category of Weil sheaves (complexes) on an $\mathbb{F}_{q^{-}}$scheme.

We upgrade the sheaf $\Delta_{0}^{e t}=\nabla_{e t}^{0}=i_{0 *}(W h)$ (cf. section 4.1.1) to a Weil sheaf $\widetilde{\Delta}_{0}^{e t}=\widetilde{\nabla}_{e t}^{0}=i_{0 *}(\widetilde{W h})$ where

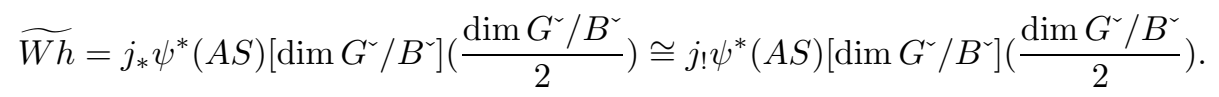

It is clear that $\widetilde{\Delta}_{0}^{e t}$ is pure of weight zero.

Then we get a functorial isomorphism $F r^{*}(\Phi(\mathcal{F})) \cong \Phi(\mathcal{F})$ for all $\mathcal{F} \in D^{G \times \mathbb{G}_{m}}(\tilde{\mathcal{N}})$. In particular, for $\mathcal{F} \in \mathcal{E}^{G \times \mathbb{G}_{m}}(\tilde{\mathcal{N}})$ the perverse sheaf $\Phi(\mathcal{F})$ is equipped with a Weil structure, thus we get a functor $\tilde{\Phi}$ from $\mathcal{E}^{G \times \mathbb{G}_{m}}(\tilde{\mathcal{N}})$ to Weil perverse sheaves on $\mathcal{F} \ell$.

Let us record the following statement, which is immediate from the definitions.

Lemma 13. a) For $\mathcal{F} \in \mathcal{E}^{G \times \mathbb{G}_{m}}(\tilde{\mathcal{N}})$ we have canonical isomorphisms $\tilde{F}(\mathcal{F}(n)) \cong$ $\tilde{F}(\mathcal{F})\left(-\frac{n}{2}\right) ; \tilde{\Phi}(\mathcal{F}(n)) \cong \tilde{\Phi}(\mathcal{F})\left(-\frac{n}{2}\right)$ (see beginning of section 2.3 for notations).

b) For $\mathcal{F}, \mathcal{G} \in D^{G \times \mathbb{G}_{m}}(\tilde{\mathcal{N}})$ the Frobenius action on

$$
\operatorname{Hom}^{\bullet}(\Phi(\mathcal{F}), \Phi(\mathcal{G}))=\operatorname{Hom}_{D^{G}(\tilde{\mathcal{N}})}^{\bullet}(\mathcal{F}, \mathcal{G})=\bigoplus_{n} \operatorname{Hom}_{D^{G \times \mathbb{G}_{m}(\tilde{\mathcal{N}})}}^{\bullet}(\mathcal{F}, \mathcal{G}(n))
$$

preserves the direct sum decomposition, and equals $q^{-n / 2}$ on the $n$-th summand.

Lemma 14. Set $J_{\lambda, n}=\tilde{F}\left(\mathcal{O}_{\lambda, n}\right)$.

Then the Weil perverse sheaf $J_{\lambda, 0}$ is pure of weight zero on the open dense stratum in its support. 
Proof. Let $V_{\lambda}$ be an irreducible representation of $G$ with extremal weight $\lambda$. Recall from $[\mathrm{AB}]$ that $Z_{\lambda}=F\left(V_{\lambda} \otimes \mathcal{O}_{\tilde{\mathcal{N}}}\right)$ is the central sheaf introduced in [G]. In particular it is a perverse sheaf. The sheaf $V_{\lambda} \otimes \mathcal{O}_{\tilde{\mathcal{N}}}$ carries an obvious $\mathbb{G}_{m}$ equivariant structure; let $\tilde{Z}_{\lambda}=\tilde{F}\left(V_{\lambda} \otimes \mathcal{O}_{\tilde{\mathcal{N}}}\right)$ be the corresponding Weil perverse sheaf. It follows from the construction of $(25)$ in $[\mathrm{AB}]$ that this Weil structure on $Z_{\lambda}$ coincides with the Weil structure defined in $[\mathrm{G}]$.

The $\mathbb{G}_{m}$ equivariant vector bundle $V_{\lambda} \otimes \mathcal{O}_{\tilde{\mathcal{N}}}$ carries a filtration with associated graded $\mathcal{O}_{\nu, 0}$, where $\nu$ runs over weights of $V_{\lambda}$. It induces a filtration on $\tilde{Z}_{\lambda}$ with subquotient $J_{\nu, 0}$. Furthermore, it is known (see [AB], [GH]) that $\mathcal{S}_{\lambda}$ is open in the support of both $J_{\lambda}$ and $Z_{\lambda}$, dense in the support of $J_{\lambda}$ and does not intersect the support of $J_{\nu}$, if $\nu$ is a weight of $V_{\lambda}$ and $\nu \neq \lambda$. It follows that $\left.\left.J_{\lambda, 0}\right|_{\mathcal{S}_{\lambda}} \cong \tilde{Z}_{\lambda}\right|_{\mathcal{S}_{\lambda}}$, and it suffices to see that the sheaf in the right hand side of the latter isomorphism has weight zero. This is clear from the construction of $\tilde{Z}_{\lambda}$ in [G].

We now define Weil perverse sheaves $\widetilde{\nabla}_{e t}^{\lambda}=\tilde{\Phi}\left(\nabla^{\lambda, \delta_{\lambda}}\right), \widetilde{\Delta}_{\lambda}^{e t}=\tilde{\Phi}\left(\Delta_{\lambda, \delta_{\lambda}}\right), \widetilde{I C}_{\lambda}=$ $\tilde{\Phi}\left(E_{\lambda, \delta_{\lambda}}\right)$ (the number $\delta_{\lambda}$ was defined in 1.4). Lemma 12 and Corollary 4 show that these are Weil perverse sheaves with underlying sheaves $\nabla^{\lambda}, \Delta_{\lambda}, I C_{\lambda}$.

Proposition 10. The Weil sheaves $\widetilde{\nabla}_{e t}^{\lambda}, \widetilde{\Delta}_{\lambda}^{e t}, \widetilde{I C}_{\lambda}$ are pure complexes of weight zero on $\mathcal{F} \ell^{\lambda}$ (the open stratum in their support).

Proof. It is enough to check the statement about $\widetilde{\Delta}_{\lambda}^{e t}$, the rest of the claim follows from this. In view of (23), restriction of $\widetilde{\Delta}_{\lambda}^{e t}$ to the open stratum $\mathcal{F} \ell^{\lambda}$ in its support is isomorphic to the convolution $\widetilde{\Delta}_{0}^{e t} * J_{\lambda, \delta_{\lambda}}$ restricted to $\mathcal{F} \ell^{\lambda}$.

The $\mathbf{G}^{\sim} \mathbf{o}$ orbit $\mathcal{F} \ell^{\lambda}$ fibers over $G^{\sim} / B^{\sim}$; Lemma 11 implies that the sheaf $i_{\lambda}^{*}\left(J_{\lambda}\right)$ is (up to shift and twist) the pull-back of the standard sheaf attached to the $B^{\sim}$

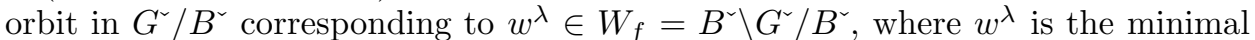
length element such that $w^{\lambda}(\lambda) \in \Lambda^{+}$; notice that $\ell\left(w^{\lambda}\right)=\delta_{\lambda}$. In view of Lemma 14 the claim follows now from the next Lemma.

For $w \in W_{f}$ let $\mathfrak{i}_{w}:\left(G^{\triangleleft} / B^{\triangleleft}\right)_{w} \hookrightarrow G^{\triangleleft} / B^{\triangleleft}$ be the embedding of the corresponding $B^{\sim}$ orbit in $G^{\sim} / B^{`}$.

Lemma 15. For $w \in W_{f}$ we have an isomorphism of Weil sheaves

$$
\widetilde{W h} * \mathfrak{i}_{w !}\left(\overline{\mathbb{Q}_{l}}[\ell(w)](\ell(w) / 2)\right) \cong \widetilde{W h}(-\ell(w) / 2) .
$$

Proof. We have $\mathfrak{i}_{w_{1} !}\left(\underline{\overline{\mathbb{Q}}}\left[\ell\left(w_{1}\right)\right]\left(\frac{\ell\left(w_{1}\right)}{2}\right)\right) * \mathfrak{i}_{w_{2}} !\left(\underline{\mathbb{Q}_{l}}\left[\ell\left(w_{2}\right)\right]\left(\frac{\ell\left(w_{2}\right)}{2}\right)\right) \cong$ $\mathfrak{i}_{w_{1} w_{2} !}\left(\overline{\mathbb{Q}_{l}}\left[\ell\left(w_{1} w_{2}\right)\right]\left(\frac{\ell\left(w_{1} w_{2}\right)}{2}\right)\right)$ provided $\ell\left(w_{1} w_{2}\right)=\ell\left(w_{1}\right)+\ell\left(w_{2}\right)$. Thus it is enough to prove the Lemma for $w=s$ a simple reflection. In this case we have a short exact sequence of perverse sheaves

$$
0 \rightarrow L_{s} \rightarrow \mathfrak{i}_{s !}\left(\underline{\overline{\mathbb{Q}_{l}}}[1](1 / 2)\right) \rightarrow \delta_{e}(-1 / 2) \rightarrow 0
$$

where $\delta_{e}$ is the skyscraper at the $B^{`}$-invariant point, and $L_{s}$ is the constant sheaf (twisted and shifted) on the closure of the orbit $\overline{\left(G^{\sim} / B^{\sim}\right)_{s}} \cong \mathbb{P}^{1}$. It is easy to see that $W h * L_{s}=0$, and $W h * \delta_{e} \cong W h$. The Lemma follows.

We are now ready to express Ext's between coherent sheaves appearing in the Positivity Lemma in terms of perverse sheaves.

Assume that $\iota_{\lambda}: x_{\lambda} \hookrightarrow \mathcal{F} \ell^{\lambda}$ is defined over $\mathbb{F}_{q}$. Then for a Weil perverse sheaf $\mathcal{F}$ on $\mathcal{F} \ell$ the graded vector spaces $\iota_{\lambda}^{*}(\mathcal{F}), \iota_{\lambda}^{!}(\mathcal{F})$ carry an action of Frobenius. Let 
$\zeta_{\lambda}$ denote the constant by which Frobenius acts on the one dimensional vector space $\iota_{\lambda}^{*}\left(\widetilde{\Delta}_{\lambda}^{e t}\right)\left[-d_{\lambda}\right]\left(-\frac{d_{\lambda}}{2}\right)$, where $d_{\lambda}=\operatorname{dim} \mathcal{F} \ell^{\lambda}$. Proposition 10 shows that $\zeta_{\lambda}$ is an algebraic number all of whose conjugates have absolute value one (in fact, it is easy to see that $\zeta_{\lambda}$ is a root of unity; we will not use this fact). Let $\iota_{\lambda}^{*}(\mathcal{F})_{[n]}^{(m)}, \iota_{\lambda}^{!}(\mathcal{F})_{[n]}^{(m)}$ denote the $q^{m / 2} \zeta_{\lambda}$ eigenspace in the $n$-th cohomology space of the corresponding (co)stalk.

Corollary 6. We have canonical isomorphisms

$$
\begin{gathered}
\operatorname{Hom}_{D^{G \times \mathbb{G}_{m}(\tilde{\mathcal{N}})}}^{i}\left(\Delta_{\lambda, n+\delta_{\lambda}}, E_{\mu, \delta_{\mu}}\right)=\iota_{\lambda}^{!}\left(\widetilde{I C}_{\mu}\right)_{\left[i+d_{\lambda}\right]}^{\left(n+d_{\lambda}\right)} ; \\
{\left[\operatorname{Hom}_{D^{G \times \mathbb{G}_{m}(\tilde{\mathcal{N}})}}^{i}\left(E_{\mu, \delta_{\mu}}, \nabla^{\lambda, \delta_{\lambda}+n}\right)\right]^{*}=\iota_{\lambda}^{*}\left(\widetilde{I C}_{\mu}\right)_{\left[-i-d_{\lambda}\right]}^{\left(n-d_{\lambda}\right)} .}
\end{gathered}
$$

Proof. The $\left(\right.$ co)stalk $\iota_{\lambda}^{*}(\mathcal{F}), \iota_{\lambda}^{!}(\mathcal{F})$ of an object $\mathcal{F} \in D^{b}\left(\mathcal{P}_{\text {asph }}\right)$ can be expressed in terms of Hom to (from) a (co)standard sheaf, see (22). Taking into account the action of Frobenius, we see that if $\mathcal{F} \in D^{b}\left(\mathcal{P}_{a s p h}\right)$ is equipped with a Weil structure, then

$$
\begin{gathered}
\operatorname{Hom}^{i}\left(\widetilde{\Delta}_{\lambda}^{e t}, \mathcal{F}\left(\frac{n}{2}\right)\right)^{F r} \cong \iota_{\lambda}^{!}(\mathcal{F})_{\left[i+d_{\lambda}\right]}^{\left(n+d_{\lambda}\right)}, \\
{\left[\operatorname{Hom}^{i}\left(\mathcal{F}, \widetilde{\nabla}_{e t}^{\lambda}\left(-\frac{n}{2}\right)\right)^{F r}\right]^{*} \cong \iota_{\lambda}^{*}(\mathcal{F})_{\left[-i-d_{\lambda}\right]}^{\left(n-d_{\lambda}\right)} .}
\end{gathered}
$$

We plug in $\mathcal{F}=\widetilde{I C}_{\mu}=\tilde{\Phi}\left(E_{\mu, \delta_{\mu}}\right)$; the claim follows then from Lemma 13.

Remark 9. The content of the Corollary can be summarized as follows. The graded components in the Ext spaces between irreducible exotic sheaves and (co) standard sheaves can be expressed using weight components of the (co)stalks of irreducible Iwahori-Whittaker sheaves. Notice shifts by $\delta_{\lambda}, \delta_{\mu}$, which come from the following three facts:

- a Wakimoto sheaf $J_{\lambda}$ is standard (extension by zero from a cell) along the generic fiber of the projection from its support to the affine Grassmannian (Lemma 11);

- dimension of this generic fiber equals $\delta_{\lambda}$ (see the proof of Proposition 10);

- the skyscraper sheaf at the zero-dimensional Schubert cell in $G^{\sim} / B^{`}$ enters the Jordan-Hölder series of the extension by zero of a weight zero sheaf on a $d$-dimensional Schubert cell with weight $-2 d$ (Lemma 15).

This shift by $\delta_{\lambda}$ matches the homological shift by $\delta_{\lambda}$ appearing in section 3 (see Theorem 3 and section 3.3); the latter is related to the homological shift arising in the weak Borel-Weil Theorem (cf. the usual Borel-Weil Theorem, which implies that, over a field of characteristic zero, $R \Gamma(G / B, \mathcal{O}(l \lambda))$ is concentrated in homological degree $\delta_{\lambda}$ for $l$ larger than the Coxeter number).

4.3. Proof of the Positivity Lemma. By Proposition $10,\left.\widetilde{I C}_{\mu}\right|_{\mathcal{F} \ell^{\mu}}$ is pure of weight zero. Hence $\widetilde{I C}_{\mu}$ is pure of weight zero by [BBD], Corollary 5.4.3. Thus the right hand side of the first (respectively, second) isomorphism in Corollary 6 vanishes for $i>n$ (respectively, $-i<n$ ) by the definition of a pure complex (cf., e.g., [BBD], Corollary 5.1.9). 


\section{REFERENCES}

[APW] Andersen, H.-H.; Polo, P.; Wen, K.X., Representations of quantum algebras, Invent. Math. 104 (1991), no. 1, 1-59.

[AB] Arkhipov, S.; Bezrukavnikov, R., Perverse sheaves on affine flags and Langlands dual group, electronic preprint math.RT/0201073, to appear in Israel J. Math.

[ABG] Arkhipov, S.; Bezrukavnikov, R.; Ginzburg, V., Quantum Groups, the loop Grassmannian, and the Springer resolution, J. Amer. Math. Soc. 17 (2004), 595-678.

[AG] Arkhipov, S.; Gaitsgory, D., Another realization of the category of modules over the small quantum group, Adv. Math. 173 (2003), no. 1, 114-143.

[BBD] Beilinson, A.; Bernstein, J.; Deligne, P., Faisceaux pervers, Astérisque, 100 (1982), 5-171.

[BBM] Beilinson, A.; Bezrukavnikov, R.; Mirković, I., Tilting exersices, Moscow Math. J. 4 (2004), no.3, 547-557.

[BG] Beilinson, A.; Ginzburg, V., Wall-crossing functors and D-modules, Represent. Theory 3 (1999), 1-31.

[BGS] Beilinson, A.; Ginzburg, V.; Soergel, W., Koszul duality patterns in representation theory, J. Amer. Math. Soc. 9 (1996), no. 2, 473-527.

[B] Bezrukavnikov, R., Perverse sheaves on affine flags and nilpotent cone of the Langlands dual group, electronic preprint, math.RT/0201256, to appear in Israel J. Math.

[B1] Bezrukavnikov, R., Perverse coherent sheaves (after Deligne), electronic preprint math.AG/0005152.

[B2] Bezrukavnikov, R., Quasi-exceptional sets and equivariant coherent sheaves on the nilpotent cone, Represent. Theory 7 (2003), 1-18.

[BMR] Bezrukavnikov, R.; Mirković, I.; Rumynin, D., Localization of modules for a semisimple Lie algebra in prime characteristic, electronic preprint, math.RT/0205144, to appear in Ann. of Math.

[BK] Bondal, A. I.; Kapranov, M. M., Representable functors, Serre functors, and reconstructions, Izv. Akad. Nauk SSSR Ser. Mat. 53 (1989), no. 6, 1183-1205, 1337; translation in Math. USSR-Izv. 35 (1990), no. 3, 519-541.

[CPS] Cline, E.; Parshall, B.; Scott, L., Abstract Kazhdan-Lusztig theories, Tohoku Math. J. (2) 45 (1993), 511-534.

[Dem] Demazure, M., A very simple proof of Bott's theorem, Invent. Math. 33 (1976), no. 3, $271-272$.

[FG] Frenkel, E., Gaitsgory, D., Local geometric Langlands correspondence and affine Kac-Moody algebras, preprint math.RT/0508382, $152 \mathrm{pp}$.

[G] Gaitsgory, D., Construction of central elements in the affine Hecke algebra via nearby cycles, Invent. Math. 144 (2001), no. 2, 253-280.

[GK] Ginzburg, V.; Kumar, S., Cohomology of quantum groups at roots of unity, Duke Math. J. 69 (1993), no. 1, 179-198.

[GH] Goertz, U.; Haines, T.J., The Jordan-Hölder series for nearby cycles on some Shimura varieties and affine flag varieties, preprint math.AG/0402143, 71 pages.

$[\mathrm{H}]$ Humphreys, J., Comparing modular representations of semisimple groups and their Lie algebras, Modular interfaces (Riverside, CA, 1995), 69-80, AMS/IP Stud. Adv. Math., 4, Amer. Math. Soc., Providence, RI, 1997.

[Lq] Lusztig, G., Introduction to quantum groups, Progress in Mathematics, 110. Birkhäuser Boston, Inc., Boston, MA, 1993.

[Lc] Lusztig, G., Cells in affine Weyl groups, IV. J. Fac. Sci. Univ. Tokyo Sect. IA Math. 36 (1989), no. 2, 297-328.

[O] Ostrik, V., On the equivariant K-theory of the nilpotent cone, Represent. Theory 4 (2000), 296-305.

[O1] Ostrik, V., Cohomological supports for quantum groups, (Russian) Funktsional. Anal. i Prilozhen. 32 (1998), no. 4, 22-34, 95; translation in Funct. Anal. Appl. 32 (1998), no. 4, 237-246 (1999)

[R] Ringel, C.M., The category of modules with good filtrations over a quasi-hereditary algebra has almost split sequences, Math. Z. 208 (1991), no. 2, 209-223.

[S] Soergel, W., Kategorie $\mathcal{O}$, perverse Garben und Moduln über den Koinvarianten zur Weylgruppe, J. Amer. Math. Soc. 3 (1990), no. 2, 421-445. 